\title{
Title: Identification and targeting of a pan-genotypic influenza A virus RNA structure that mediates packaging and disease
}

Abstract: Currently approved anti-influenza drugs target viral proteins, are subtype limited, and are challenged by rising antiviral resistance. To overcome these limitations, we sought to identify a conserved essential RNA secondary structure within the genomic RNA predicted to have greater constraints on mutation in response to therapeutics targeting this structure. Here, we identified and genetically validated an RNA stemloop structure we termed PSL2, which serves as a packaging signal for genome segment PB2 and is highly conserved across influenza A virus (IAV) isolates. RNA structural modeling rationalized known packaging-defective mutations and allowed for predictive mutagenesis tests. Disrupting and compensating mutations of PSL2's structure give striking attenuation and restoration, respectively, of in vitro virus packaging and 
mortality in mice. Antisense Locked Nucleic Acid oligonucleotides (LNAs) designed against

PSL2 dramatically inhibit IAV in vitro against viruses of different strains and subtypes, possess a

high barrier to the development of antiviral resistance, and are equally effective against

oseltamivir carboxylate-resistant virus. A single dose of LNA administered 3 days after, or 14

days before, a lethal IAV inoculum provides $100 \%$ survival. Moreover, such treatment led to the development of strong immunity to rechallenge with a ten-fold lethal inoculum. Together, these results have exciting implications for the development of a versatile novel class of antiviral therapeutics capable of prophylaxis, post-exposure treatment, and "just-in-time" universal vaccination against all IAV strains, including drug-resistant pandemics.

One Sentence Summary: Targeting a newly identified conserved RNA structure in the

11 packaging signal region of influenza segment PB2 abrogates virus production in vitro and dramatically attenuates disease in vivo.

\section{Introduction:}

Influenza A virus (IAV) is a segmented RNA virus that causes major morbidity and mortality worldwide. Current antiviral therapies target viral proteins that frequently mutate, rendering many such therapies inadequate ${ }^{1-4}$. Despite a breadth of knowledge about the viral lifecycle, knowledge of the RNA secondary structure of the genome is limited. Recent research on other RNA viruses has revealed genomic RNA to be capable of playing many important roles in viral lifecycles beyond merely encoding amino acid sequences, suggesting that viral RNA structural elements could be promising therapeutic targets ${ }^{5,6}$. To the extent these RNA structural elements are both essential and highly conserved, these features could reduce the degree of freedom for mutations that are compatible with virus function. This in turn, could translate into a high barrier for resistance to therapeutics designed to disrupt these RNA structures. In IAV, 
1 genome packaging is one such critical juncture in which RNA structure might serve a central

The IAV genome consists of eight single-stranded negative-sense viral RNA (vRNA) segments that encode a minimum of 14 known viral proteins ${ }^{7}$. The vRNA, together with nucleoprotein (NP) and the heterotrimeric polymerase complex, comprised of PB2, PB1, and PA proteins, forms the complete viral ribonucleoprotein (vRNP) $)^{8,9}$. To be fully infectious, IAV virions must incorporate at least one of each segment's vRNP ${ }^{10,11}$. Current paradigm supports a selective packaging method whereby the eight vRNPs are selected in a hierarchal, segmentdependent manner mediated by unique, segment-specific packaging signals present in the terminal and central coding regions of each segment that allow for discrimination between the $\mathrm{vRNAs}^{9,12,13}$. Each vRNP interacts with at least one other partner to form a supramolecular complex ${ }^{14}$ likely maintained by intersegment RNA-RNA and/or protein-RNA interactions hypothesized to guide the packaging process ${ }^{10,15}$. The mechanism mediating this selection and arrangement, however, is poorly understood. Curiously, packaging signals exist in regions of high nucleotide conservancy that strongly suppress synonymous codon usage ${ }^{16-18}$. Conservation of primary sequence beyond what is required for protein coding suggests the potential for maintenance of RNA structures possessing biological functionalities. Certain synonymous mutations within the polymerase gene, PB2, not only affect its own packaging, but also the incorporation of other segments ${ }^{11,13,16}$. We hypothesized that PB2's dominant role in the packaging process might be facilitated by non-protein elements encoded by the PB2 RNA, including structured RNA elements. To test this hypothesis, we first solved the RNA secondary structure within PB2 that mediates packaging. We then genetically validated this structure's critical role in the viral life cycle in vitro and IAV pathogenesis in vivo. Finally, we show proof- 
1 of-concept for a new class of antiviral therapeutics that can efficiently disrupt packaging and completely prevent and treat otherwise lethal disease in vivo, as well enable the development of strong functional immunity, with a high barrier to resistance.

\section{Results}

\section{SHAPE-characterization of IAV segment PB2 packaging signal identifies conserved structure}

To search for structured RNA domains, we first applied selective 2'-hydroxyl acylation analyzed by primer extension "SHAPE"19 and computational modeling to IAV segment PB2 genomic vRNA. In vitro transcribed full-length (-)-sense PB2 vRNA from strain A/Puerto Rico/8/1934 (H1N1) "PR8" was folded in solution ${ }^{20}$ and interrogated using an electrophilic SHAPE reagent that preferentially reacts with nucleotides existing in flexible, single-stranded states $^{19}$ (Fig. 1). This analysis revealed that much of the 2341-nt vRNA is largely unstructured (Supplementary Fig.1a), consistent with other bioinformatics studies that found higher potential for RNA secondary structure conservation in the (+)-sense over the (-)-sense RNA for all segments, including PB2 ${ }^{17,21}$. However, these previous studies did not analyze the terminal coding regions (TCR), and instead stopped 80 nucleotides short of the PB2 5' TCR's end.

SHAPE-guided modeling suggested several areas in this terminal region that contain stable RNA secondary structures, most notably a stem-loop motif, named herein as Packaging Stem-Loop 2 (PSL2) (Fig. 1a and Supplementary Fig.1b, nucleotides 34-87). This region included a set of nucleotides that were previously implicated in segment PB2 packaging through mutational analysis via an unidentified mechanism (Fig. 1a,b, see circled nucleotides, and Supplementary

Table 1) ${ }^{13,16,18}$. Supporting the hypothesis that these prior mutations act through disruption of PSL2 structure, SHAPE analysis of the mutants yielded different conformations that all abrogated the wild-type PSL2 structure (Fig. 1c, and Supplementary Fig. 2). The 60-nucleotide 
1 region encompassing PSL2 displays near 100\% sequence conservation at the single nucleotide level between representative seasonal as well as pandemic strains of different subtypes and species origins (Fig. 1d, Supplementary Fig. 1c). Further analysis revealed that this high degree of conservation extends to all known IAV isolates available in public databases (Supplementary Fig.1d), suggesting the existence of a strict biologic requirement to maintain an intact PSL2 structure. To exclude the possibility that differing downstream sequences within PB2 vRNA could alter the secondary structure of PSL2, we explored PSL2's structural conservancy by performing SHAPE on full-length wild-type PB2 vRNAs from a variety of IAV strains and subtypes, including the highly pathogenic avian H5N1 and pandemic $1918 \mathrm{H} 1 \mathrm{~N} 1$ strains. Despite the presence of two diverging nucleotides within the stem-loop and significant modeling of PB2 RNA across these diverse species and subtypes (Fig. 1e).

\section{Mutate-and-Map strategy validates PSL2 structure and predicts novel packaging mutants}

To further test the SHAPE analysis of the PSL2 RNA structure and to uncover additional informative mutations needed for in vivo tests, we applied multidimensional chemical mapping methods to the PSL2 segment. Mutate-and-Map ( $\left.\mathrm{M}^{2}\right)$ analysis couples systematic mutagenesis with high-throughput chemical mapping to produce accurate base-pair inferences and interactions of RNA domains ${ }^{22}$. By sequentially mutating RNA one nucleotide at a time with its Watson-Crick complement and measuring the impact this mutagenesis has on chemical reactivity, pair-wise correlations between close and distant residues can be established. First, $\mathrm{M}^{2}$ measurements confirmed disruption of the chemical reactivity pattern upon systematic mutation of each PSL2 stem residue, including changes at nucleotides previously identified to be relevant for PB2 packaging (Fig. 2, see noted fields) ${ }^{13,16}$. Automated computational analysis based on 
1 these $\mathrm{M}^{2}$ data recovered the SHAPE-guided PSL2 structure with high confidence (Fig. 2,

2 Supplementary Figs. 2 and 3), further validating our structural model. Second, as predictive

3 tests, we designed compensatory mutations to restore base pairings - albeit not the native

4 sequence - in the wild-type stem-loop structure that were disrupted by the initial packagingdefective mutations (Supplementary Fig. 4). These mutation-rescue variants indeed restored the

PSL2 SHAPE pattern, providing in vitro validation of the modeled structure at base-pair resolution and suggesting sequence variants to test the role of PSL2 structure in vivo.

To test whether the PSL2 stem-loop structure observed in solution was relevant to virus packaging in the cellular milieu, the same nine synonymous mutations reported by Gog et al. (2007) and Marsh et al. (2008) (Fig. 1a,b and Supplementary Table 1) as well as four new synonymous mutations characterized by $\mathrm{M}^{2}$ analysis (Fig. 2a,b) were cloned into pDZ plasmids containing the PR8 PB2 gene ${ }^{13,16,18}$. The packaging efficiencies of the nine previously known mutants, now in the PR8 background, were comparable to those originally described in the WSN33 virus ${ }^{13}$ (Fig. 1b and Supplementary Table 1). Of these, mutants m55c, m757, m745, and $\mathrm{m} 744 \mathrm{~b}$, were predicted to show the most significant impairment based on their location within PSL2's stem regions (Fig. 1a-c and Supplementary Fig. 2). In contrast, published mutations that have no effect on PB2 packaging (e.g. m731) mapped to the unstructured apical loop or fell outside of PSL2 and did not alter its structural integrity (Supplementary Fig. 5a), while mutations with minor effects to virus packaging showed only minor alterations to the structure (Supplementary Fig. 5b) ${ }^{13}$. The three novel synonymous mutants (m74-1, m74-2, and m68) identified by $\mathrm{M}^{2}$-analysis as having a significant effect on in vitro PSL2 structure (Fig. 2a, see green-marked nucleotides) showed significant loss in PB2 packaging, whereas mutation sites that resulted in negligible change in SHAPE reactivity compared to wild-type PSL2 (e.g., m56), 
1 gave wild-type-like packaging efficiency levels (Fig. 2b). The strong correlation of structure disruption with in cellulo packaging efficiency observed across these mutants supports a role of PSL2 structure in virus packaging.

\section{Compensatory mutation pairs restore PSL2 structure, rescue packaging in vitro and in vivo}

disease mutations designed to restore the wild-type stemloop structure destroyed by the packagingdefective mutations (Fig. 2c, Supplementary Fig. 4) were cloned into PR8 pDZ plasmids to generate mutant rescue viruses. The compensatory mutations rescued not only the virus packaging for segment PB2 (Fig. 2d), but also other segments previously reported to be affected by the deleterious mutations, consistent with the proposed hierarchal role of PB2 in IAV packaging (Supplementary Fig. 6a) ${ }^{11-13}$. In addition to recovering PB2 packaging, the compensatory mutations gave complete or near-complete rescue of the virus titer loss caused by the defective mutations (Fig. 2e and Supplementary Fig. 6b). Some non-synonymous compensatory mutations were able to restore PB2 packaging better than others (m745-comp and m55c-comp, compared to m757-comp). This possibly reflects incomplete restoration of PB2

17 protein function through exogenous addition (Fig. 2d, e and Supplementary Fig. S6) since for non-synonymous mutations, we also expressed WT PB2 protein to mitigate the possibility of any impairment in PB2 protein function.

The most incisive test of PSL2 structure came from packaging experiments that did not require supplemental wild-type PB2 protein addition. Computational enumeration and multidimensional mutation-rescue $\mathrm{e}^{23}\left(\mathrm{M}^{2} \mathrm{R}\right)$ experiments were performed in order to identify additional successful PSL2-defective and compensatory mutant pairs (Fig. 3a, b and 
1 Supplementary Fig. 7). Successful mutation-rescue was defined when each single mutation

2 alone resulted in disrupting the SHAPE-mapped wild-type PSL2 structure, while the double

3 compensatory mutations recovered wild-type PSL2 structure (Fig. 3a, b see boxed

4 electropherograms). Although most discovered successful partners required non-synonymous

5 changes, we discovered a single mutation-rescue pair of substitutions that were both

6 synonymous, obviating wild-type PB2 protein addition (Fig. 3b, c and Supplementary Figs. 7, 8

7 and 9). Making each mutation alone (m52 and m65) resulted in severe packaging defects and

8 virus titer loss exceeding $4 \log _{10}$ - an extreme impairment beyond what has been previously

9 reported $^{13,16}$ (i.e. 1-2 $\log _{10}$ ) for packaging-defective viruses (Figs. 3d, e and Supplementary

10 Figs. 6 and 8). When introduced together into a doubly mutated m52/65-compensatory strain that

11 restored PSL2 structure, albeit with an altered sequence, the compensatory mutations restored

12 both packaging efficiency and virus titer to wild-type levels. These data provided unambiguous

13 evidence for the PSL2 52-65 RNA base pair in influenza A packaging. In order to ensure that

14 any loss or subsequent rescue of virus packaging was not due to defects in replication or

15 translation caused by these synonymous mutations, each of the PB2 mutation-rescue mutants

16 were tested in a transfection-based replicon assay to assess both the ability of the generated PB2

17 protein mutants to function as part of the polymerase complex, as well as to test the ability of the

18 PB2 mutant vRNA to be replicated. All mutant PB2 proteins and vRNAs were produced at

19 comparable wild-type levels (Supplementary Figs. 10 and 11).

20 To test the relevance of the PSL2 structure in an in vivo model, 6-8 week old BALB/c

21 mice were intranasally instilled with either wild-type or mutant PR8 viruses harboring point

22 mutations predicted to disrupt or restore PSL2 structure. Mice infected with the PSL2-disrupting

23 mutations - m745 mutant strain (20\% packaging efficiency) or the severely packaging-defective 
1 single mutant virus, m52 (<4\% packaging efficiency) — showed reduced or no clinical signs of

2 illness, respectively, either in weight loss or survival as compared to the PBS control (Fig. 3f, g).

3 Remarkably, inclusion of compensatory mutations that restore PSL2 structure rescued virus

4 pathogenicity: animals infected with m52/65-comp and m745-comp, displayed comparable mortality profiles as mice infected with wild-type PR8 (Fig. 3f, g). To the best of our knowledge, these are the first data indicating that packaging-defective viruses are attenuated in vivo and a genomic IAV RNA secondary structure mediates influenza disease progression. Given the strong evolutionary conservation of the predicted PSL2 structure across different IAV subtypes, strains, and host species isolates (Fig. 1d, e and Supplementary Fig. 1c, d), we postulated that therapeutics directed against this structure could possess broad-spectrum antiviral activity against all IAV subtypes and strains.

\section{Therapeutic design and antisense targeting of PSL2 structure inhibits IAV infection in vitro}

To explore the therapeutic potential of targeting PSL2-mediated virus packaging, nine antisense oligonucleotides (ASO) with modified locked nucleic acid (LNA) bases containing

15 phosphorothioate internucleoside linkages ${ }^{24}$ were designed against PSL2 residues in a manner predicted to disrupt various aspects of the overall RNA secondary structure of the motif and their

17 effect on inhibition of virus production was determined (Fig. 4a). Two of the designed LNAs, LNA8 and LNA9, are identical in sequence to LNA6 and LNA7, respectively, but possess 6-7 unmodified (non-locked) DNA nucleotide "gapmers" optimized for RNase-H activation that can

20 degrade RNA in RNA-DNA hybrids ${ }^{25}$. First, to assess the impact that LNA binding has on PSL2

21 RNA secondary structure, toeprinting and SHAPE chemical mapping were performed on PB2

22 vRNA in the presence of the LNAs. Sequences encoded in LNAs 6-9, corresponding to binding 
1 sites on the right $3^{\prime}$ side of the stemloop structure (Fig. 4a), exhibited the greatest ability to bind and disrupt the wild-type PSL2 structure (Supplementary Fig. 12).

To test the antiviral potential of LNA-mediated targeting of PSL2 across different IAV subtypes, MDCK cells were pre-treated with $100 \mathrm{nM}$ of each LNA for 4 hours prior to infection with either the wild-type PR8 (H1N1) virus or the tissue culture-adapted A/Hong Kong/8/1968 (HK68) (H3N2) virus. Forty-eight hours post-infection, the supernatants were collected, and virus production was measured by plaque assays (Fig. 4b). As predicted by our mutational and LNA chemical mapping experiments (Fig. 2, Supplementary Fig. 12), LNAs directed against only the top loop of PSL2 (LNA1, LNA4), and LNAs solely targeting the 3' base of PSL2 (e.g., LNAs 3 and 5) had minimal effect on viral titer. In contrast, nucleotide coverage of both the top loop and middle bulge by LNA6 resulted in greater than $2 \log _{10}$ titer deficits for PR8 (Fig. 4a, b). LNA8, the RNase-H activated copy of LNA6, produced even greater antiviral activity against both viruses of up to $3 \log s_{10}$. Most strikingly, LNA9, the RNase-H activated copy of LNA7,

possessed the strongest antiviral capacity, dropping virus production by over $4 \log s_{10}$ and 3

$\operatorname{logs}_{10}$ against PR8 and HK68, respectively. While LNA9 could be clearly visualized in cells harboring vRNPs (Supplementary Fig. 13), no off-target effect of LNA9 on steady state levels of viral protein, vRNA, cRNA, or cellular toxicity after 24 hours was observed (Supplementary Figs. 14, 15, and 16). The potent antiviral activity of these LNAs corroborate the results from the LNA chemical mapping experiment, indicating that other compounds that can similarly disrupt the PSL2 structure are likely to possess antiviral activity.

Having identified a potent candidate LNA, we next investigated the treatment timecourse and concentration parameters of LNA9's antiviral activity. MDCK cells were treated with varying concentrations of LNA9 at either 2 or 4 hours pre-infection or, alternatively, 2 or 4 hours 
1 post-infection with wild-type PR8 virus. Cells pre-treated with the LNA had the most potent antiviral response (greater than $4 \log s_{10}$ ) and displayed strong virus inhibition (greater than 2

$\left.3 \operatorname{logs}_{10}\right)$ even at the lowest tested concentration $(1 \mathrm{nM})($ Fig. $4 \mathbf{c})$. There was a trend towards decreasing antiviral activity as the time post-infection treatment increased, but even at the latest tested time point of addition, greater than $2 \operatorname{logs}_{10}$ suppression of viral titer was achieved. Similar efficacy was seen in the presence of high MOI (Supplementary Fig. 17). To probe whether LNA-mediated virus inhibition resulted in loss of PB2 packaging, cells were treated with 100 nM of LNA9 or scrambled LNA and then infected with PR8 virus. Isolated vRNA from packaged virions were then analyzed for virus packaging by qPCR. Similar to the mutational studies, LNA9 treatment resulted in a dramatic loss of PB2 packaging compared to controls (Fig. 4d). To test the hypothesis that therapeutic targeting of the PSL2 motif would possess broadspectrum antiviral activity, cells were pretreated with $100 \mathrm{nM}$ of LNA9 or scrambled LNA prior to infection with more clinically relevant human strains, including the 2009 pandemic "swine" (pH1N1) virus. Both the modern H3N2 and pH1N1 viruses were highly sensitive to LNA9 treatment, showing inhibition at levels greater than or equal to results seen against PR8 or HK68 (Fig. 4e).

\section{In vitro selection of IAV variants under escalating drug pressure}

Our designed LNAs are directed against a highly conserved genomic RNA target (Fig.

1c, Supplementary Fig. 1c, d) that appears to have strong biologic constraints against viable viral mutants. We hypothesized that this additional level of mutational constraint would provide a higher barrier to the development of resistance for therapeutics directed against PSL2. To test this hypothesis, we determined the susceptibility of wild-type PR8 virus to LNA9 under conditions designed to promote the development of resistance over serial virus passaging (Fig. 
5a). In parallel, we performed analogous experiments using the neuraminidase inhibitor (NAI) oseltamivir carboxylate (OSLT, Tamiflu ${ }^{\mathrm{TM}}$ ). OSLT had a starting EC $\mathrm{E}_{50}$ of $4.1 \mathrm{nM}$ against PR8 at passage 1 of drug treatment. After seven virus passages in the presence of escalating drug concentrations, the $\mathrm{EC}_{50}$ of OSLT leapt to $100 \mu \mathrm{M}$ - a greater than 20,000x fold increase (Fig. 5b). In comparison, after 10 passages of virus in the presence of LNA9, the $\mathrm{EC}_{50}$ held in the picomolar range of 16 to $22 \mathrm{pM}$. To date, we have yet to be able to select for viral mutations capable of generating resistance to LNA9. To test if LNA9 is efficacious against drug-resistant viruses, a drug-resistant mutant of A/WSN/33 (H1N1) virus was generated using a reverse

genetic virus rescue system that mutated the NA gene to contain the H275Y mutation (N1

numbering system), known to confer resistance to NAIs. Against this NAI-resistant virus, LNA9 maintained the same potency and efficacy it exerted against wild-type, while high-level resistance to OSLT was confirmed, with an $\mathrm{EC}_{50}$ of $53 \mu \mathrm{M}$ (Fig. 5 c-e). This result extends the apparent therapeutic capabilities of LNA9 and provides strong support for potential therapeutic treatment of NAI-resistant viruses with PSL2-targeting LNAs.

\section{PSL2-targeted LNAs protect mice from lethal IAV infections}

As a proof-of-concept to assess the in vivo efficacy of prophylactic LNA treatment against PSL2, BALB/c mice were intranasally (I.N.) administered a single $20 \mu \mathrm{g}$ dose of LNA9 or scrambled LNA one day, or three days, prior to infection with a lethal dose of PR8 virus. The untreated control mice experienced dramatic weight loss and were humanely sacrificed by days 5 and 6. In contrast, a single dose of LNA9 was completely protective when administered one day, and even three days, prior to viral infection, and showed significantly reduced virus titer in the lungs ( $>2.5 \log _{10}$ virus reduction) compared to the scrambled control at $72 \mathrm{hrs}$ post-infection (Fig. 6a, Supplementary Fig. 18). In addition to the well-known benefits of LNA antisense 
1 gapmer technology (e.g., high target binding affinity, RNase-H cleavage activity, and high nuclease resistance conferred by the thiolated phosphate backbone), LNA ASOs have also been reported to show dramatic, long-lasting effects (even $>1$ month) after the last administered dose in a variety of disease models ${ }^{26-29}$. We hypothesized that PSL2-targeted LNAs might similarly possess long-term prophylactic effects. week (Day -7) prior to infection with a lethal dose of IAV (Fig. 6b). While 100\% of untreated mice succumbed to the infection, $70 \%$ of the LNA9 one-week pretreatment group were protected from lethality. To determine if our therapeutics can be further optimized for improved efficacy, we fine-tuned the delivery formulation as well as reconsidered the LNA target region. While LNA9 targets nucleotides in the lower 3' stem of PSL2, our mutational analyses suggest the importance of the 52-65 nucleotide pair in the upper stem (Fig. 4). In fact, none of LNAs1-9

13 fully covered this region, so we hypothesized that additional LNA design against these nucleotides might enhance efficacy (Fig. 4a). The newly designed LNA14 fully covers the top biological confirmation of this, mice treated with LNA14 were fully protected when given a

18 single dose one week prior to infection with a lethal dose of IAV (Fig. 6e). Pushing the prophylactic window even further, we again increased the LNA dosage (40 $\mu \mathrm{g})$ and administered 20 a single LNA14 treatment to mice two-weeks (Day -14) prior to IAV infection. While the mock21 treated mice were sacrificed between days 7 and 8 due to severe disease, the entire LNA14- 
1 undetectable disease symptoms and reduced weight loss compared to controls (Fig. $6 \mathbf{f}-\mathbf{h}$, Supplementary Table 2).

Anti-PSL2 LNA treatment protects mice from lethal infection in part by attenuating the virus and reducing disease progression; however, because mice do display symptoms, albeit minor, we hypothesized that the resulting highly attenuated infection might be sufficient to enable mice receiving prophylactic LNA treatment to develop an effective immunization against a secondary infection through production of long-term immunity. To test this hypothesis, mice $(n=7)$ from the one-week LNA14 pretreatment surviving cohort (Fig. 6e) were challenged alongside age-matched, naïve controls, sixty-five days post-primary infection with ten times the mouse lethal dose $\left(10 \mathrm{LD}_{100}\right)$ of IAV (Fig. 6i). The resulting secondary challenge had no effect on weight, clinical score, or survival of mice from the LNA14 pretreatment group (a total of 72 days since treatment, 65 days since primary infection), while the age-matched controls presented with rapid disease and were humanely sacrificed by Day 6 post-challenge infection (Fig. 6j-I).

After demonstrating PSL2-targeted LNA efficacy in prophylactic models, we next sought to establish its potential as a post-infection therapeutic. Due to the rapid onset of symptoms and illness in IAV infections, FDA-approved IAV therapeutics are most challenged when administered after $48 \mathrm{hrs}$ of disease onset ${ }^{30}$. To assess the ability of anti-PSL2 LNAs to treat IAV well after an infection has been established, we sought to deliver our LNAs in a way most likely to be administered therapeutically to hospitalized, severely ill patients: by I.V. injection. Mice infected with a lethal dose of PR8 virus $(n=10)$ were treated with either LNA14, LNA9, Scrambled LNA, or vehicle control by intravenous injection on day 3 post-infection (Day +3$)$ when mice typically become noticeably ill, to simulate a severe, hospitalized infection. While mice treated with vehicle and scrambled controls rapidly succumbed to the infection, 
1 approximately $65 \%$ of mice treated with LNA9 survived lethal infection, and all mice in the LNA14-treated cohort survived (Fig. 6m).

\section{Discussion}

We describe here the discovery and characterization of an RNA stemloop structure, PSL2, that serves as a packaging signal for genome segment PB2, which is conserved across influenza A isolates. Knowledge of PSL2's RNA secondary structure not only can explain previous fortuitously discovered packaging-defective mutations, but also enabled the rational design of mutations with more potent disruption of packaging. Moreover, compensatory mutations that restore PSL2's structure (but not primary sequence) rescue virus packaging and titer loss in vitro, thus providing strong genetic validation of the importance of PSL2's RNA secondary structure for influenza virus packaging. Extending these findings in vivo, PSL2 disrupting and compensating mutants give striking attenuation and restoration, respectively, of mortality in mice - confirming PSL2's importance not only to the viral life cycle, but also for influenza-mediated disease. Antisense locked nucleic acids (LNAs) designed to disrupt PSL2 structure dramatically inhibit IAV in vitro against viruses of different strains and subtypes, exhibit a high barrier to the development of resistance, and are equally effective against wildtype and NAI-resistant viruses. In vivo, intranasal dosing of LNAs resulted in potent antiviral efficacy and prevented mortality in mice, even with a single dose administered two weeks prior to infection with a lethal IAV inoculum. In a therapeutic model, an anti-PSL2 LNA provided complete protection from death when administered three days post-infection with a lethal IAV

21 dose. Moreover, the PSL2-targeting LNAs not only provided full protection against lethal IAV 22 infection, but also enabled the surviving mice to develop vigorous immunity. Together, these results have exciting implications for the development of a novel class of pan-genotypic anti- 
1 IAV therapies for prophylaxis of, treatment of established, and "just-in-time" universal vaccination against, an IAV infection.

Historically, it was assumed that IAV RNA secondary structures would be inaccessible in vRNP complexes due to the association between the vRNA and NP; however, local RNA structures can remain recognizable as substrates. For example, bound RNA in vRNP complexes are still accessible to treatment by RNases, and unprotected vRNA regions are sufficient for hybridization to complementary cDNA fragments ${ }^{31,32}$. Furthermore, some findings suggest the presence of NP may actually increase vRNA base accessibility and thus might enable intermolecular RNA-RNA interactions, rather than impede them ${ }^{33}$. We therefore hypothesized that local structural motifs may be exposed at the surface of the vRNPs, and could be enabling RNA-RNA or RNA-protein interactions that guide the packaging process.

This hypothesis led to the discovery of a novel stemloop structure, PSL2, in the 5' terminal-coding region of IAV segment PB2 genomic vRNA known to be important for virus packaging. Previously-defined, as well as more informed and potent newly designed, packagingdefective mutations ${ }^{13,16}$ in this region destroyed PSL2 structure and yielded packaging-defective viruses that were attenuated in vivo, while compensatory mutations designed to restore the structure rescued virus packaging in vitro and restored lethality in an in vivo mouse model. Given the large body of work characterizing IAV packaging signals, we were initially surprised that the critical synonymous mutation sites, m52 and m65, had gone undiscovered or unremarked by prior studies. However, despite previous efforts that sequentially mutated the PSL2 region that included these nucleotide regions ${ }^{13}$, their mutational strategy suffered from lack of knowledge of the stemloop structure in two important ways. First, the previously described nt65 mutation was C65U, which still produces a basepaired G-U in the PSL2 stem, generating a structurally 
1 tolerated mutation. In contrast, our m65 (C65A) mutation does not base pair with the opposing guanine nucleotide and shows severe structure disruption (Fig. 3, Supplementary Fig. 7). Second, to our knowledge no previous work mutated the m52 nucleotide, but rather mutated its adjacent neighbor (nt C50A), which lies in an exposed bulge of PSL2. There are six possible permissible synonymous changes to the arginine amino acid (ARG 755) that covers nucleotide 52, almost all leading to a structurally permissible change. Without information about the PSL2 structure, this critical base pair and its relevance to PB2 packaging could easily be overlooked. Importantly, we found that restoration of PSL2 structure through compensatory viral lineages and subtypes to be swapped during co-infection leading to the production of novel

13 viruses. This propensity towards viral reassortment in both the form of antigenic drift, which leads to new strains that result in our need for yearly vaccine redesign, and viral shift, which pandemics, necessitates a certain level of conservancy in the packaging signal regions of each segment to allow for reassortment between different viruses. The sequence region in segment PB2 that contains the PSL2 stemloop is highly conserved across IAV subtypes, strains, and host species restricted viruses, and likely reflects a strict biologic requirement for its preservation. SHAPE analysis of this region confirmed maintenance of the PSL2 structure between seasonal as well as pandemic viruses of different subtypes and host origins, suggesting that this structural element could be a novel broad-spectrum therapeutic target. Although our structural studies are based on in vitro RNA templates, we expect that rapidly emerging improvements in RNA 
1 structure probing technologies will enable a more detailed view of PSL2's RNA structure within cells and in the context of the vRNP complex.

Recent studies have confirmed and further expanded on the role that RNA structure plays in the IAV lifecycle ${ }^{34-43}$. These include the findings that regions of low NP-binding fall within packaging signals and are likely to contain RNA structures ${ }^{39,40}$, the continued emergence of new RNA-RNA interactions between segments during the packaging process ${ }^{34}$, the discovery that specific nucleotide residues are important to the formation of precise vRNP structures critical to genome packaging ${ }^{43}$, the uncovering of new RNA secondary structures in the MX gene and their roles in the production of infectious particles ${ }^{35,36}$, and excitingly, the first global RNA structure of the IAV genome mapped in cells ${ }^{41}$, to name a few. As the technology to detect and monitor RNA structures and their interactions in the context of viral infection grows, we anticipate many new discoveries of the unique roles these structures, including PSL2, play in IAV and how they interact to guide the packaging and virus production process.

In our present study, we have demonstrated that LNA-mediated targeting of the PSL2 structure has potent antiviral activity in vitro across divergent IAV subtypes, and importantly, protects against lethal IAV infection in vivo after as little as a single intranasal dose given two weeks before infection, or three days post-infection. Moreover, mice treated with LNA prior to inoculation with a lethal IAV dose show robust long-lasting immunity against high titer virus rechallenge. One of the limits to the currently approved IAV vaccine strategy is its inadequate production of long-term humoral immunity, with recent research suggesting that viral infection is far superior to vaccination in the protective efficacy against heterosubtypic circulating virus ${ }^{44-46}$. Given the potential for long-lasting prophylactic protection after a single dose of LNA14, combined with the universality of the pan-genotypic PSL2 target, it is attractive to speculate on 
1 the clinical use of anti-PSL2 LNAs as a "just-in-time" universal vaccine strategy. In addition, because traditional vaccines take weeks to provide full protection, a co-administered single dose of our LNAs could provide protection during this vulnerability window.

The use of RNA-based therapeutics to treat disease is a rapidly growing field. The advent of LNA technology allows for the design of antisense oligonucleotides (ASO) that possess greater biologic capabilities than their siRNA and non-LNA base counterparts, both in terms of in vivo stability and target specificity. Several ASO-mediated therapeutics have now gained FDA-approval for use in humans ${ }^{47,48}$, and there are an ever-growing number of promising candidates currently in clinical trials ${ }^{49,50}$. Recently, an siRNA against respiratory syncytial virus in lung transplant recipients has shown success in a Phase $2 b$ trial, where the siRNA was

11 delivered nasally via a nebulizer ${ }^{51}$. We envision a similar method of treatment and path to the clinic for PSL2-targeted therapies, like LNA14 against IAV, although intravenous administration

13 for severe hospitalized patients may be a complementary path. Moreover, analogous strategies can now be contemplated for rapidly identifying and targeting critical RNA secondary structures

15 in a wide range of viruses for which no effective therapies currently exist. Importantly, incorporating the targeted virus' RNA secondary structure into the design of antiviral LNAs

17 allowed us to achieve far greater inhibition than with ASOs designed against the same viral genomic sequence but which relied only on primary nucleotide sequence homology for their $19 \operatorname{design}^{52}$.

Oseltamivir (Tamiflu $\left.{ }^{\mathrm{TM}}\right)$ is the most widely used and stockpiled neuraminidase inhibitor

21 (NAI) on the market. Like all NAIs, OSLT requires a conformational rearrangement in the viral

22 NA protein to accommodate the drug. Any mutations in the NA protein that affect this 
1 H275Y mutation is most associated with OSLT resistance. Selection for resistance mutations is

2 of particular concern with IAV, especially in immunocompromised populations, where it was

3 shown that the rapid selection of the H275Y mutation in an immunocompromised patient lead to

4 clinical failure of the last-resort NAI drug, peramivir, suggesting that the selection for multi-drug resistant viruses in immunocompromised hosts may be more common than previously believed ${ }^{1}$.

6 Moreover, IAV has demonstrated the ability to acquire resistance to OSLT in unexpected ways ${ }^{53}$.

7 This, together with the recent spread of OSLT-resistant and NAI-resistant viruses in

8 circulation $^{54,55}$, indicates that we may need to reevaluate our usage of NAIs as a whole and are in 9 urgent need of orthogonal anti-IAV therapeutics.

11 replication by targeting the endonuclease function of the viral polymerase complex, was

12 approved by the FDA in late 2018. It is the first novel flu treatment to receive FDA approval 13 since the clearance of NAIs oseltamivir and zanamivir in $1999^{56}$. Like NAIs, however, BXM 14 targets a viral protein, making the development of drug resistance a concern. Indeed, in its first 15 year of use, reports of viruses with reduced susceptibilities to BXM were isolated from cell 16 cultures, from patients in clinical trials, and from adults infected with $\mathrm{A} / \mathrm{H} 1 \mathrm{~N} 1$, as well as 17 pediatric patients infected with $\mathrm{A} / \mathrm{H} 3 \mathrm{~N} 2^{57,58}$. The rapid emergence of resistance mutations and the ease at which they arise in both the circulating virus subtypes, constitute a clear warning for

19 the drug's clinical efficacy. Here, we demonstrate that PSL2-targeted LNAs can possess a high barrier to the development of antiviral resistance, and that LNAs can retain their same high

21 potency against NAI-resistant strains. Such discovery of new viral targets and the development

22 of new classes of antivirals is imperative to reduce the adverse impact current and future 23 pandemics can have on human health.

\section{References and Notes:}




\footnotetext{
1. Memoli, M.J., Hrabal, R.J., Hassantoufighi, A., Eichelberger, M.C. \& Taubenberger, J.K. Rapid selection of oseltamivir- and peramivir-resistant pandemic H1N1 virus during therapy in 2 immunocompromised hosts. Clin Infect Dis 50, 1252-1255 (2010).

2. Hai, R., et al. Influenza A(H7N9) virus gains neuraminidase inhibitor resistance without loss of in vivo virulence or transmissibility. Nature communications 4, 2854 (2013).

3. Hayden, F.G. \& de Jong, M.D. Emerging influenza antiviral resistance threats. J Infect Dis 203, 6-10 (2011).

4. Liu, X., et al. Poor responses to oseltamivir treatment in a patient with influenza A (H7N9) virus infection. Emerging microbes \& infections 2, e27 (2013).

5. Parsons, J., et al. Conformational inhibition of the hepatitis C virus internal ribosome entry site RNA. Nat Chem Biol 5, 823-825 (2009).

6. Romero-Lopez, C. \& Berzal-Herranz, A. Unmasking the information encoded as structural motifs of viral RNA genomes: a potential antiviral target. Rev Med Virol 23, 340-354 (2013).

7. $\quad$ Palese, P.S., M. L. Fields Virology, (Lippincott Williams \& Wilkins, 2007).

8. Compans, R.W., Content, J. \& Duesberg, P.H. Structure of the ribonucleoprotein of influenza virus. J Virol 10, 795-800 (1972).

9. Hutchinson, E.C., von Kirchbach, J.C., Gog, J.R. \& Digard, P. Genome packaging in influenza A virus. $J$ Gen Virol 91, 313-328 (2010).

10. Noda, T. \& Kawaoka, Y. Structure of influenza virus ribonucleoprotein complexes and their packaging into virions. Rev Med Virol 20, 380-391 (2010).

11. Muramoto, Y., et al. Hierarchy among viral RNA (vRNA) segments in their role in vRNA incorporation into influenza A virions. J Virol 80, 2318-2325 (2006).

12. Gao, Q., et al. The influenza A virus PB2, PA, NP, and M segments play a pivotal role during genome packaging. J Virol 86, 7043-7051 (2012).

13. Marsh, G.A., Rabadan, R., Levine, A.J. \& Palese, P. Highly conserved regions of influenza a virus polymerase gene segments are critical for efficient viral RNA packaging. J Virol 82, 2295-2304 (2008).

14. Fournier, E., et al. A supramolecular assembly formed by influenza A virus genomic RNA segments. Nucleic Acids Res 40, 2197-2209 (2012).

15. Gavazzi, C., et al. An in vitro network of intermolecular interactions between viral RNA segments of an avian H5N2 influenza A virus: comparison with a human H3N2 virus. Nucleic Acids Res 41, 1241-1254 (2013).

16. Gog, J.R., et al. Codon conservation in the influenza A virus genome defines RNA packaging signals. Nucleic Acids Res 35, 1897-1907 (2007).

17. Moss, W.N., Priore, S.F. \& Turner, D.H. Identification of potential conserved RNA secondary structure throughout influenza A coding regions. Rna 17, 991-1011 (2011).

18. Liang, Y., Huang, T., Ly, H. \& Parslow, T.G. Mutational analyses of packaging signals in influenza virus PA, PB1, and PB2 genomic RNA segments. $J$ Virol 82, 229-236 (2008).

19. Wilkinson, K.A., Merino, E.J. \& Weeks, K.M. Selective 2'-hydroxyl acylation analyzed by primer extension (SHAPE): quantitative RNA structure analysis at single nucleotide resolution. Nature protocols 1, 1610-1616 (2006).

20. Pang, P.S., Elazar, M., Pham, E.A. \& Glenn, J.S. Simplified RNA secondary structure mapping by automation of SHAPE data analysis. Nucleic Acids Res 39, e151 (2011).

21. Priore, S.F., Moss, W.N. \& Turner, D.H. Influenza A virus coding regions exhibit host-specific global ordered RNA structure. PloS one 7, e35989 (2012).

22. Kladwang, W. \& Das, R. A mutate-and-map strategy for inferring base pairs in structured nucleic acids: proof of concept on a DNA/RNA helix. Biochemistry 49, 7414-7416 (2010).

23. Tian, S., Cordero, P., Kladwang, W. \& Das, R. High-throughput mutate-map-rescue evaluates SHAPEdirected RNA structure and uncovers excited states. Rna 20, 1815-1826 (2014).

24. Vester, B. \& Wengel, J. LNA (locked nucleic acid): high-affinity targeting of complementary RNA and DNA. Biochemistry 43, 13233-13241 (2004).

25. Kurreck, J., Wyszko, E., Gillen, C. \& Erdmann, V.A. Design of antisense oligonucleotides stabilized by locked nucleic acids. Nucleic Acids Res 30, 1911-1918 (2002).

26. Straarup, E.M., et al. Short locked nucleic acid antisense oligonucleotides potently reduce apolipoprotein B mRNA and serum cholesterol in mice and non-human primates. Nucleic Acids Res 38, 7100-7111 (2010).

27. Staedel, C., et al. Inhibition of Gastric Tumor Cell Growth Using Seed-targeting LNA as Specific, Longlasting MicroRNA Inhibitors. Molecular therapy. Nucleic acids 4, e246 (2015).
} 
28. Javanbakht, H., et al. Liver-Targeted Anti-HBV Single-Stranded Oligonucleotides with Locked Nucleic Acid Potently Reduce HBV Gene Expression In Vivo. Molecular therapy. Nucleic acids 11, 441-454 (2018).

29. Hillebrand, F., et al. Gymnotic Delivery of LNA Mixmers Targeting Viral SREs Induces HIV-1 mRNA Degradation. Int J Mol Sci 20(2019).

30. CDC.gov. Influenza Treatment. (Centers for Disease Control and Prevention, National Center for Immunization and Respiratory Diseases (NCIRD), cdc.gov, 2019).

31. Klumpp, K., Ruigrok, R.W. \& Baudin, F. Roles of the influenza virus polymerase and nucleoprotein in forming a functional RNP structure. Embo J 16, 1248-1257 (1997).

32. Coloma, R., et al. The structure of a biologically active influenza virus ribonucleoprotein complex. PLoS Pathog 5, e1000491 (2009).

33. Baudin, F., Bach, C., Cusack, S. \& Ruigrok, R.W. Structure of influenza virus RNP. I. Influenza virus nucleoprotein melts secondary structure in panhandle RNA and exposes the bases to the solvent. Embo $J$ 13, 3158-3165 (1994).

34. Gilbertson, B., et al. Influenza NA and PB1 Gene Segments Interact during the Formation of Viral Progeny: Localization of the Binding Region within the PB1 Gene. Viruses 8(2016).

35. Kobayashi, Y., et al. Computational and molecular analysis of conserved influenza A virus RNA secondary structures involved in infectious virion production. RNA Biol 13, 883-894 (2016).

36. Spronken, M.I., et al. A compensatory mutagenesis study of a conserved hairpin in the $\mathrm{M}$ gene segment of influenza A virus shows its role in virus replication. RNA Biol 14, 1606-1616 (2017).

37. Dawson, W.K., Lazniewski, M. \& Plewczynski, D. RNA structure interactions and ribonucleoprotein processes of the influenza A virus. Briefings in functional genomics 17, $402-414$ (2018).

38. Ferhadian, D., et al. Structural and Functional Motifs in Influenza Virus RNAs. Front Microbiol 9, 559 (2018).

39. Williams, G.D., et al. Nucleotide resolution mapping of influenza A virus nucleoprotein-RNA interactions reveals RNA features required for replication. Nature communications $\mathbf{9}, 465$ (2018).

40. Bolte, H., Rosu, M.E., Hagelauer, E., Garcia-Sastre, A. \& Schwemmle, M. Packaging of the Influenza Virus Genome Is Governed by a Plastic Network of RNA- and Nucleoprotein-Mediated Interactions. $J$ Virol 93(2019).

41. Dadonaite, B., et al. The structure of the influenza A virus genome. Nat Microbiol (2019).

42. Shafiuddin, M. \& Boon, A.C.M. RNA Sequence Features Are at the Core of Influenza A Virus Genome Packaging. J Mol Biol (2019).

43. Takizawa, N., et al. Local structural changes of the influenza A virus ribonucleoprotein complex by single mutations in the specific residues involved in efficient genome packaging. Virology 531, 126-140 (2019).

44. Osterholm, M.T., Kelley, N.S., Sommer, A. \& Belongia, E.A. Efficacy and effectiveness of influenza vaccines: a systematic review and meta-analysis. Lancet Infect Dis 12, 36-44 (2012).

45. Sridhar, S., et al. Cellular immune correlates of protection against symptomatic pandemic influenza. Nat Med 19, 1305-1312 (2013).

46. Kim, J.H., et al. Prior infection with influenza virus but not vaccination leaves a long-term immunological imprint that intensifies the protective efficacy of antigenically drifted vaccine strains. Vaccine 34, 495-502 (2016).

47. Biogen. U.S. FDA Approves Biogen's SPINRAZA ${ }^{\text {TM }}$ (nusinersen), The First Treatment for Spinal Muscular Atrophy. in Press Release, Vol. 2017 (Biogen Press Release, 2016).

48. Nature.com. FDA approves antisense cholesterol drug. in Nature News Blog, Vol. 2017 (2013).

49. Coelho, T., et al. Safety and efficacy of RNAi therapy for transthyretin amyloidosis. N Engl J Med 369, 819-829 (2013).

50. Fitzgerald, K., et al. Effect of an RNA interference drug on the synthesis of proprotein convertase subtilisin/kexin type 9 (PCSK9) and the concentration of serum LDL cholesterol in healthy volunteers: a randomised, single-blind, placebo-controlled, phase 1 trial. Lancet 383, 60-68 (2014).

51. Gottlieb, J., et al. ALN-RSV01 for prevention of bronchiolitis obliterans syndrome after respiratory syncytial virus infection in lung transplant recipients. J Heart Lung Transplant 35, 213-221 (2016).

52. Giannecchini, S., Clausi, V., Nosi, D. \& Azzi, A. Oligonucleotides derived from the packaging signal at the 5 ' end of the viral PB2 segment specifically inhibit influenza virus in vitro. Arch Virol 154, 821-832 (2009).

53. Bloom, J.D., Gong, L.I. \& Baltimore, D. Permissive secondary mutations enable the evolution of influenza oseltamivir resistance. Science 328, 1272-1275 (2010). 

54. Besselaar, T.G., et al. Widespread oseltamivir resistance in influenza A viruses (H1N1), South Africa. Emerg Infect Dis 14, 1809-1810 (2008).
55. Hurt, A.C., et al. Characteristics of a widespread community cluster of H275Y oseltamivir-resistant A(H1N1)pdm09 influenza in Australia. J Infect Dis 206, 148-157 (2012).
56. Mifsud, E.J., Hayden, F.G. \& Hurt, A.C. Antivirals targeting the polymerase complex of influenza viruses. Antiviral research 169, 104545 (2019).
57. Takashita, E., et al. Detection of influenza A(H3N2) viruses exhibiting reduced susceptibility to the novel cap-dependent endonuclease inhibitor baloxavir in Japan, December 2018. Euro Surveill 24(2019).
58. Takashita, E., et al. Influenza A(H3N2) virus exhibiting reduced susceptibility to baloxavir due to a polymerase acidic subunit I38T substitution detected from a hospitalised child without prior baloxavir treatment, Japan, January 2019. Euro Surveill 24(2019).
59. Hoffmann, E., Neumann, G., Kawaoka, Y., Hobom, G. \& Webster, R.G. A DNA transfection system for generation of influenza A virus from eight plasmids. Proc Natl Acad Sci U S A 97, 6108-6113 (2000).
60. Szretter, K.J., Balish, A.L. \& Katz, J.M. Influenza: propagation, quantification, and storage. Current protocols in microbiology Chapter 15, Unit 15G 11 (2006).
61. Marsh, G.A., Hatami, R. \& Palese, P. Specific residues of the influenza A virus hemagglutinin viral RNA are important for efficient packaging into budding virions. J Virol 81, 9727-9736 (2007).
62. Kawakami, E., et al. Strand-specific real-time RT-PCR for distinguishing influenza vRNA, cRNA, and mRNA. J Virol Methods 173, 1-6 (2011).
63. Mortimer, S.A. \& Weeks, K.M. Time-resolved RNA SHAPE chemistry: quantitative RNA structure analysis in one-second snapshots and at single-nucleotide resolution. Nature protocols 4, 1413-1421 (2009).

64. Akbari, A., et al. Improved DNA fragment length estimation in capillary electrophoresis. Electrophoresis 29, 1273-1285 (2008).

65. Pang, P.S., et al. Structural map of a microRNA-122: hepatitis C virus complex. J Virol 86, 1250-1254 (2012).

66. Deigan, K.E., Li, T.W., Mathews, D.H. \& Weeks, K.M. Accurate SHAPE-directed RNA structure determination. Proc Natl Acad Sci U S A 106, 97-102 (2009).

67. De Rijk, P., Wuyts, J. \& De Wachter, R. RnaViz 2: an improved representation of RNA secondary structure. Bioinformatics 19, 299-300 (2003).

68. Kladwang, W., VanLang, C.C., Cordero, P. \& Das, R. A two-dimensional mutate-and-map strategy for non-coding RNA structure. Nature chemistry 3, 954-962 (2011).

69. Kladwang, W., Cordero, P. \& Das, R. A mutate-and-map strategy accurately infers the base pairs of a 35nucleotide model RNA. RNA 17, 522-534 (2011).

70. Cordero, P., Kladwang, W., VanLang, C.C. \& Das, R. A mutate-and-map protocol for inferring base pairs in structured RNA. in RNA Folding (Methods in Molecular Biology) (ed. Waldsich, C.) in press. http://arxiv.org/abs/1301.7734 (2013).

71. Mortimer, S.A. \& Weeks, K.M. A fast-acting reagent for accurate analysis of RNA secondary and tertiary structure by SHAPE chemistry. J Am Chem Soc 129, 4144-4145 (2007).

72. Yoon, S., et al. HiTRACE: high-throughput robust analysis for capillary electrophoresis. Bioinformatics 27, 1798-1805 (2011).

73. Kim, H., Cordero, P., Das, R. \& Yoon, S. HiTRACE-Web: an online tool for robust analysis of highthroughput capillary electrophoresis. Nucleic Acids Research 41, W492-W498 (2013).

74. Kim, J., et al. A robust peak detection method for RNA structure inference by high-throughput contact mapping. Bioinformatics 25, 1137-1144 (2009).

75. Kladwang, W., et al. Standardization of RNA chemical mapping experiments. Biochemistry 53, 3063-3065 (2014).

76. Mathews, D.H., et al. Incorporating chemical modification constraints into a dynamic programming algorithm for prediction of RNA secondary structure. Proc Natl Acad Sci U S A 101, 7287-7292 (2004).

77. Darty, K., Denise, A. \& Ponty, Y. VARNA: Interactive drawing and editing of the RNA secondary structure. Bioinformatics 25, 1974-1975 (2009).

78. Cordero, P., Lucks, J.B. \& Das, R. An RNA Mapping DataBase for curating RNA structure mapping experiments. Bioinformatics 28, 3006-3008 (2012). 
1 Acknowledgements: We thank Hong Jin and her team at MedImmune for the kind donation of chicken eggs and technical guidance. Additional thanks go to Phil Pang for initial study

3 brainstorming. The work was supported in part by a Mona M. Burgess Stanford BIO-X Interdisciplinary Graduate Fellowship, the National Institutes of Health (NIH) Graduate Training Grant 5T32AI007328-24, NIH research grants R56A1111460, U19A1109662, RO1AI132191, a Harrington Scholar Innovator Grant, and W81XWH1810647 from USAMRAA, Department of National Institute of Allergy and Infectious Diseases, National Institutes of Health. The data presented in this manuscript are tabulated in the main paper and in the supplementary materials. Chemical mapping datasets for mutate-and-map and mutation/rescue experiments have been deposited at the RNA Mapping Database (http://rmdb.stanford.edu). R.J.H. and J.S.G. conceived and designed the study. R.J.H., M.E., L.B., K.N., A.X. and S.T. performed the experiments. R.J.H., M.E., S.T., and L.B. analyzed the data. M.E. and T.A. provided expertise and assistance Trademark Office. 


\section{Materials and Methods}

Cells and viruses. HEK 293 T and MDCK cells (NLB-2) were obtained from American Type Culture Collection 'ATCC' (Manasass, VA) and were maintained in Dulbecco's modified Eagle's medium with 10\% fetal bovine serum and penicillin-streptomycin (Gibco). All cell lines used in this report were routinely checked for mycoplasma contamination (MycoAlert Mycoplasma Detection Kit, Lonza) and were authenticated by the respective vendors. Wild-type influenza A/PR/8/34 (PR8) H1N1 virus (ATCC-VR-95) and the tissue-culture adapted PR8 virus (ATCC-VR-1469) were purchased from ATCC. PR8 mutant viruses were generated using an eight-plasmid reverse genetic system as previously described ${ }^{59}$. Tissue-cultured adapted influenza A/Hong Kong/8/68 (HK68) H3N2 virus (ATCC-VR-1679), A/Virginia/ATCC6/2012 (H3N2) virus (ATCC-VR-1811), A/Virginia/ATCC1/2009TC (H1N1) virus (ATCC-VR-1736), and A/Wisconsin/33 (H1N1) virus (VR-1520) were purchased from ATCC. A/California/4/2009 (pH1N1) virus was kindly gifted by Elena Govorkova from St. Jude Children's Research Hospital (Memphis, USA). Viruses were grown and amplified in 10-day-old specific-pathogenfree research grade chicken embryos at $35^{\circ} \mathrm{C}$ (Charles River Laboratories; SPAEAS).

Plasmid constructs and cloning. Plasmids were used containing the wild-type PB2 segments from influenza viruses A/PuertoRico/8/34 (H1N1) [PR8], A/New York/470/2004 (H3N2) [NY470], A/New York/312/2001 (H1N1) [NY312], A/Brevig Mission/1/1918 (H1N1) [1918], A/California/04/2009 (H1N1) [CA09], and A/Vietnam/03/2004 (H5N1) [VN1203]. For the generation of PR8 packaging mutant vRNA, we utilized a Stratagene QuickChange XL sitedirected mutagenesis kit (Stratagene) for mutagenesis of a pDZ plasmid containing the PB2 gene of PR8 ${ }^{59}$. Sequences of each mutated construct were confirmed by automated sequencing. The 8plasmid pBD rescue system for A/WSN/33 (H1N1) was kindly donated by Andrew Mehle. The 
1 H275Y NA mutant was generated by QuickChange mutagenesis from the bidirectional pBD

2 plasmids, as described above.

3 Reverse genetics and virus titrations. Recombinant A/Puerto Rico/8/34 (PR8) virus and recombinant $\mathrm{A} / \mathrm{WSN} / 33$ (WSN) virus were generated using eight-plasmid reverse genetic systems $^{59}$. Briefly, $10^{6}$ cells of a 293T/MDCK co-culture were Lipofectamine ${ }^{\mathrm{TM}} 3000$ (Invitrogen ${ }^{\mathrm{TM}}$ ) transfected with $1 \mu \mathrm{g}$ of one of each of the eight segments contained within plasmids that utilize a bidirectional dual Pol I/II promoter system for the simultaneous synthesis of genomic vRNA and mRNA. For rescue of compensatory PB2 mutant viruses where a nonsynonymous change was required, a wild-type PB2 protein expression plasmid (Pol II) was cotransfected during virus rescue. Supernatants were collected 24 hours post-transfection. PR 8 rescue viruses were then inoculated into the allantoic cavities of 10-day-old chicken embryos. WSN rescue viruses were passaged subsequent times on MDCK cells. Rescue of recombinant viruses was assessed by hemagglutination activity. Each newly rescued virus was further plaque titered and mutations were confirmed by sequencing of mutated genes. Plaque assays were carried out on confluent MDCK cells as described previously ${ }^{60}$. Hemagglutination (HA) assays were carried out in 96-well round-bottomed plates at room-temperature, using $50 \mu 1$ of virus dilution and $50 \mu 1$ of a $0.5 \%$ suspensions of turkey red blood cells (LAMPIRE ${ }^{\circledR}$ Biological Laboratories) in phosphate-buffered saline (PBS).

Isolation of packaged vRNAs. To analyze packaged vRNA for PR8 mutated viruses, 10-dayold eggs were inoculated with approximately 1000 PFU of recombinant virus and incubated for 72 hours. Allantoic fluid was harvested, and supernatant was dual-clarified by low-speed centrifugation. Clarified supernatant was then layered on a $30 \%$ sucrose cushion and ultracentrifuged at 30,000 RPM for 2.5 hours (Beckman Rotor SW41). Pelleted virus was 
resuspended in PBS and TRIzol (Invitrogen) extracted. Precipitated vRNA was resuspended in a final volume of $20 \mu \mathrm{l}$ of $10 \mathrm{mM}$ Tris- $\mathrm{HCl}(\mathrm{pH} 8.0)$ and stored at $-80^{\circ} \mathrm{C}$.

Virus supernatant from LNA-treated cells was harvested 48hpi and subjected to lowspeed centrifugation at 1000 RPM then 10,000 RPM. Isolation continued as indicated above. qPCR analysis of packaged vRNAs. Approximately $200 \mathrm{ng}$ of extracted vRNA was reverse transcribed using a universal 3' primer (5'-AGGGCTCTTCGGCCAGCRAAAGCAGG) and Superscript III reverse transcriptase (RT) (Invitrogen). The RT product was diluted approximately 10,000-fold and used as a template for quantitative PCR (qPCR). Separate PCRs were then carried out as previously described ${ }^{61}$ with segment-specific primers. The $10 \mu 1$ reaction mixture contained $1 \mu \mathrm{l}$ of diluted RT product, a $0.5 \mu \mathrm{M}$ primer concentration, and SYBR Select Master Mix (Applied Biosystems) that included SYBR GreenER dye, $200 \mu \mathrm{M}$ deoxynucleoside triphosphates, heat labile UDG, optimized SYBR Green Select Buffer, and AmpliTaq DNA polymerase UP enzyme. Relative vRNA concentrations were determined by analysis of cycle threshold values, total vRNA amount within a sample was normalized to the level of HA vRNA, and then percentages of incorporation were calculated relative to the levels of wild-type vRNA packaging. Viral packaging results represent the averaged levels of vRNA incorporation \pm standard deviations derived from two independent virus purifications, with vRNA levels quantified in triplicate.

Strand-specific RT-qPCR. MDCK cells transfected with 1 mM LNA-9 or scrambled LNA were infected with PR8 virus at an MOI of 0.124 hours post transfection. Eight hours post infection total cellular RNA was extracted in Trizol reagent (Invitrogen) and the RNA was purified using the Direct-Zol RNA mini-prep (Zymo Research) according to the manufacturer protocol. Reverse transcription and qPCR were performed according to ${ }^{62}$. cDNAs of the 
influenza viral RNA (vRNA) and complementary viral RNA (cRNA) were synthesized with tagged primers to add an 18-20 nucleotide tag that was unrelated to the influenza virus at the $5^{\prime}$ end (cRNAtag; 5'-GCT AGC TTC AGC TAG GCA TC-3', vRNAtag; 5'-GGC CGT CAT GGT GGC GAA T-3'). Hot-start reverse transcription with the tagged primer was performed as described in Kawakami et al., 2011 using saturated trehalose. A $5.5 \mu$ mixture containing 200 ng

of total RNA sample and 10 pmol of tagged primer were heated for $10 \mathrm{~min}$ at $65^{\circ} \mathrm{C}$, chilled immediately on ice for $5 \mathrm{~min}$, and then heated again at $60^{\circ} \mathrm{C}$. After $5 \mathrm{~min}, 14.5 \mu \mathrm{l}$ of preheated reaction mixture [4 $\mu$ l First Strand buffer $(5 \times$, Invitrogen), $1 \mu \mathrm{l} 0.1 \mathrm{M}$ dithiothreitol, $1 \mu \mathrm{dNTP}$ $\operatorname{mix}(10 \mathrm{mM}$ each), $1 \mu \mathrm{l}$ Superscript III reverse transcriptase (200 U/ $\mu 1$, Invitrogen $), 1 \mu \mathrm{RNasin}$ Plus RNase inhibitor (40 U/ $\mu \mathrm{l}$, Promega) and $6.5 \mu$ l saturated trehalose] was added and incubated for 1 h. Real-time PCR (qPCR) was performed with PowerUp SYBR Green SuperMix (Applied Biosystems) on a BIORAD CFX96 Real-Time System. Seven microliters of a ten-fold dilution of the cDNA was added to the qPCR reaction mixture [10 $\mu$ l SYBR Green SuperMix $(2 \times), 1.5$ $\mu \mathrm{l}$ forward primer $(10 \mu \mathrm{M}), 1.5 \mu \mathrm{l}$ reverse primer $(10 \mu \mathrm{M})]$. The cycle conditions of qPCR were $95^{\circ} \mathrm{C} 10 \mathrm{~min}$, followed by 40 cycles of $95^{\circ} \mathrm{C} 15 \mathrm{sec}$ and $60^{\circ} \mathrm{C}$ for $1 \mathrm{~min}$. qPCR primers were: PR8 segment 1 (PB2) cRNA, Forward: 5'-TCC ACC AAA GCA AAG TAG AAT GC-3'; Reverse: 5'-GCT AGC TTC AGC TAG GCA TCA GTA GAA ACA AGG TCG TTT TTA AAC-3'. PR8 segment 1(PB2) vRNA, Forward: 5'-GGC CGT CAT GGT GGC GAA TAG ACG AAC AGT CGA TTG CCG AAG C-3', Reverse: 5'-AGT ACT CAT CTA CAC CCA TTT TGC-3'. PR8 segment 4 (HA) cRNA, Forward: 5'-CTG TAT GAG AAA GTA AAA AGC C-3', Reverse: 5'-GCT AGC TTC AGC TAG GCA TCA GTA GAA ACA AGG GTG TTT TTC-3'. PR8 segment 4 (HA) vRNA, Forward: 5'-GGC CGT CAT GGT GGC GAA TAG GAT GAA CTA TTA CTG GAC CTT GC-3', Reverse: 5'-TCC TGT AAC CAT CCT CAA TTT GGC-3'. 
1 Animals. All animal studies were performed in accordance with the National Institutes of Health

2 Guidelines for the Care and Use of Laboratory Animals and approved by the Stanford University

3 Administrative Panel on Laboratory Animal Care. Six to eight Healthy age-matched female

4 BALB/c mice (Jackson Laboratories, Bar Harbor ME) were randomly separated into groups for

5 infection/treatment or used as uninfected/non-treated controls. Treatment groups were not

6 blinded to the investigators. Mice were identified with tag numbers throughout the experiment.

7 In vivo infection. Mice were lightly anesthetized with isoflurane and intranasally infected with

8 with $50 \mu \mathrm{l}$ of virus preparation at a concentration of 1000 PFU for virus packaging mutant

9 experiments and 900 PFU for LNA treatment experiments. Weights and clinical scores were

10 assessed daily, and animals were humanely sacrificed when a clinical score of 5 was recorded

11 (see Supplementary Table 2 for clinical score determination). Kaplan-Meier survival curves were

12 generated using GraphPad Prism.

13 In vivo antiviral assays. 'In vivo-ready' LNAs were custom designed and ordered from Qiagen

14 (formally Exiqon). For intranasal delivery, in vivo-ready LNA was mixed in complexes with In

15 vivo-JetPEI ${ }^{\circledR}$ transfection reagent (Polyplus) according to manufacturer's protocol to the

16 indicated final concentration in $50 \mu \mathrm{l}$ of $5 \%$ glucose solution. Mice were then lightly

17 anesthetized with isoflurane and $50 \mu \mathrm{l}$ of the solution was delivered intranasally. For retro-orbital

18 delivery: 'In vivo-ready' LNA was mixed in complexes with In vivo-JetPEI® transfection

19 reagent (Polyplus) according to manufacturer's protocol to the indicated final concentration in

$20200 \mu \mathrm{l}$ of $5 \%$ glucose solution. Mice were then anesthetized, and the solution was delivered by

21 retro-orbital injection.

22 Locked Nucleic Acid (LNA) design and preparation. Oligonucleotides containing locked

23 nucleic acids (LNA) were custom synthesized from Exiqon (Vedbaek, Denmark), and later by 
1 IDT. Capitalized letters denote LNA. Lowercase letters denote typical (non-locked) DNA

nucleotides. All oligonucleotides contain phosphorothioate internucleoside linkages. LNA 8 and

9 were designed as LNA gapmers to contain a stretch of 6-7 DNA nucleotides optimized for

RNAse-H recruitment. Sequences of all LNAs are shown below.

LNA 1: 5' AccAaaAGaaT 3'

LNA 2: 5' TggCcATcaaT 3'

LNA 3: 5' TagCAtActtA 3'

LNA 4: 5' CCAAAAGA 3'

LNA 5: 5' CATACTTA 3'

LNA 6: 5' CagaCaCGaCCaaAA 3'

LNA 7: $\mathbf{5}^{\prime}$ TAcTtaCTgaCagCC $\mathbf{3}^{\prime}$

LNA 8: 5' AGAcacgaccaaAAG 3' -with RNase-H activity

LNA 9: 5' TACTtactgacaGCC 3' -with RNase-H activity

LNA14: 5' CGACcaaaagaATTC 3' -with RNase-H activity

Scramble LNA (negative control): 5' AACACGTCTATACGC 3'

In vitro LNA antiviral assays. LNAs were reconstituted in RNAse-free water at $100 \mu \mathrm{M}$ stock solutions, aliquoted and stored at $-20{ }^{\circ} \mathrm{C}$ prior to single-use. Lipofectamine $3000 \AA$ (Life

Technologies) was used to transfect LNA into cells at indicated concentrations per manufacturer's protocol. For prophylactic antiviral assays, $10^{6} \mathrm{MDCK}$ cells were plated in 6well plates 24 hours prior to being transfected with the indicated LNA. Cells were then infected at the indicated time points with $0.01 \mathrm{MOI}$ of PR8 (H1N1) or HK68 (H3N2) virus. For postinfection therapeutic antiviral assessment, MDCK cells were infected with PR8 or HK68 prior to LNA transfection as described above. Forty-eight hours post-infection, supernatant was collected, and viral titer was determined by plaque assay in triplicate.

LNA-treatment and packaging efficiency determination. Briefly, T75 flasks of $80 \%$ confluent MDCK cells were transfected with $100 \mathrm{nM}$ of scrambled LNA, LNA9, or mock untreated by 
1 Lipofectamine 3000 transfection, according to manufacturer's protocol. Twelve hours post transfection, cells were infected with $0.01 \mathrm{MOI}$ of wild-type TC-adapted PR8 virus. After 1 hour,

3 virus was removed and cells were washed with PBS. Forty-eight hours post-infection supernatants were collected, and RNA was isolated as described in isolation of packaged vRNAs and assay methods.

In vitro drug selection. LNA9 selection: $80-90 \%$ confluent MDCK cells in 12 -well plates were transfected in duplicate with a starting concentration of $0.01 \mathrm{nM}\left(\sim 1 / 2 \mathrm{EC}_{50}\right) \mathrm{LNA} 9$ for Passage 1 by Lipofectamine transfection (see above). Twelve hours post-transfection, cells were washed with PBS and infected with 0.01 MOI of wild-type PR8 virus. After 1 hour incubation at $37^{\circ} \mathrm{C}$, cells were washed and virus growth media was added. Cells were incubated until $50 \% \mathrm{CPE}$ was

11 evident (48-72 hours). Virus supernatant was harvested, low-speed centrifuge clarified,

12 aliquoted, plaque titered and stored at $-80^{\circ} \mathrm{C}$. The virus supernatant was then continuously

13 serially passaged in the presence of escalating concentrations of LNA9 (0.01 nM to $100 \mathrm{nM})$. If

14 no CPE was evident, drug concentration was lowered and added virus concentration was

15 increased until $50 \%$ CPE occurred. OSLT selection: confluent MDCK cells in 12-well plates were infected with 0.01 MOI of PR8 virus. After adsorption for an hour, cells were washed with PBS, and OSLT (Sigma Aldrich Cat. No. Y0001340) was added to virus growth media at a

18 starting concentration of $1 \mathrm{nM}\left(\sim 1 / 2 \mathrm{EC}_{50}\right)$. Drug selection proceeded as described above, with

19 escalating concentrations of $\operatorname{OSLT}(0.01 \mathrm{nM}$ to $250 \mu \mathrm{M})$ at each subsequent passage.

20 EC $_{50}$ determination. For LNA9, the 50\% effective concentration $\left(\mathrm{EC}_{50}\right)$ was defined as the 21 concentration of drug effective in reducing the percent of virus titer to $50 \%$ of that for the no-

22 drug control. In brief, the $\mathrm{EC}_{50}$ was determined by seeding $5 \times 10^{5} \mathrm{MDCK}$ cells in each well of a 23 12-well plate and incubating overnight at $37^{\circ} \mathrm{C}$ under $5 \% \mathrm{CO}_{2}$. Cells were then transfected with 
$1 \quad$ LNA9 as described above at concentrations from $0.01 \mathrm{nM}$ to $10 \mu \mathrm{M}$. Plates were incubated at $37^{\circ} \mathrm{C}$ for 12 hours prior to infection with $0.01 \mathrm{MOI}$ of wild-type PR8, serially passaged LNAtreated virus, WSN33 wild-type or WSN33 H275Y NAI-resistant virus. Forty-eight hours postinfection, supernatants were collected, centrifuge clarified, aliquoted and stored at $-80^{\circ} \mathrm{C}$. The viral titer for each drug dilution was performed by plaque assay in duplicate. The $\mathrm{EC}_{50}$ was the concentration of LNA9 yielding a percent titer of $50 \%$ of that without drug.

For OSLT, the $\mathrm{EC}_{50}$ was defined as the concentration of drug reducing the total percentage of plaques to $50 \%$ of that for the no-drug control, determined by plaque reduction assay $^{1}$. Briefly, confluent MDCK cells in 12-well plates were infected with approximately 100 PFU of wild-type PR8, serially passaged OSLT-treated virus, WSN33 wild-type or WSN33 H275Y NAI-resistant virus and incubated for 1 hour at $37^{\circ} \mathrm{C}$. Cells were then washed with PBS, and a 50:50 mix of $1 \%$ agarose to $2 \mathrm{x}$ virus growth DMEM containing varying concentrations of drug $(0.1 \mathrm{nM}$ to $1 \mathrm{mM})$ was added to the cells. Plates were harvested 72 hours later, stained with crystal violet, and plaques were counted. The $\mathrm{EC}_{50}$ was the concentration of OSLT reducing the total percentage of plaques to $50 \%$ of that without drug. All results were plotted in GraphPad Prism to generate $\mathrm{EC}_{50}$ curves.

In vitro transcription of full-length vRNA. For each wild-type isolate (PR8, 1918, VN1203, NY470, NY312, and CA09) and PR8 packaging mutant clones, PB2 cDNA was amplified from plasmid using segment-specific primers under a T7 promoter. Amplified cDNA was gel-purified using an Invitrogen DNA gel kit. vRNAs were then produced by in vitro transcription, using T7MEGAscript kit. vRNAs for SHAPE were purified by MEGAclear (Thermofisher, cat. no. AM1908) with purity and length verified by capillary electrophoresis. 
Sf-SHAPE analysis of full-length IAV vRNA. In vitro transcribed PB2 vRNA was folded (100

$\mathrm{mM} \mathrm{NaCl} ; 2.5 \mathrm{mM} \mathrm{MgCl} ; 65^{\circ} \mathrm{C} \mathrm{x} \mathrm{1',} 5^{\prime}$ cooling at room temperature, $37^{\circ} \mathrm{C}$ for $\left.20-30^{\prime}\right)$ in 100 mM HEPES, $\mathrm{pH}=8$. 2' acylation with $\mathrm{NMIA}^{19}$ and reverse transcription (RT) primer extension were performed at $45^{\circ} \mathrm{Cx} 1^{\prime}, 52^{\circ} \mathrm{C}$ x $25^{\prime}, 65^{\circ} \mathrm{C}$ x $5^{\prime}$, as previously described ${ }^{63}$. $6 \mathrm{FAM}$ was used for all labeled primers (primer sequences available upon request). Exceptions to these protocols were as follows: (i) RNA purification after acylation was performed using RNA C\&C columns (Zymo Research), rather than ethanol precipitation; (ii) before and after SHAPE primer buffer was added, the mixture was placed at room temperature for 2-5 min, which enhanced RT transcription yields significantly; (iii) DNA purification was performed using Sephadex G-50 size exclusion resin in $96-$ well format then concentrated by vacuum centrifugation, resulting in a more significant removal of primer; and (iv) 2 pmol RNA was used in ddGTP RNA sequencing reactions.

The ABI 3100 Genetic Analyzer (50 cm capillaries filled with POP6 matrix) was set to the following parameters: voltage $15 \mathrm{kV}, \mathrm{T}=60^{\circ} \mathrm{C}$, injection time $=15 \mathrm{~s}$. The GeneScan program was used to acquire the data for each sample, which consisted of purified DNA resuspended in 9.75 $\mu \mathrm{l}$ of Hi-Di formamide, to which $0.25 \mu \mathrm{l}$ of ROX 500 internal size standard (ABI Cat. 602912) was added. PeakScanner parameters were set to the following parameters: smoothing=none; window size $=25$; size calling=local southern; baseline window=51; peak threshold $=15$. Fragments 250 and 340 were computationally excluded from the ROX500 standard ${ }^{64}$. The data from PeakScanner were then processed into SHAPE data by using FAST (fast analysis of SHAPE traces), a custom algorithm developed in our lab ${ }^{20}$. FAST automatically corrects for signal differences due to handling errors, adjusts for signal decay, and converts fragment length to nucleotide position, using a ddGTP ladder as an external sizing standard and the local 
1 Southern method ${ }^{20,65}$. This algorithm embedded in the RNAstructure program is freely available at http://med.stanford.edu/glennlab/download.html. RNAstructure parameters: slope and intercept parameters of 2.6 and $-0.8 \mathrm{kcal} / \mathrm{mol}$, were initially tried, as suggested ${ }^{66}$; however, we found that smaller intercepts closer to $0.0 \mathrm{kcal} / \mathrm{mol}$ (e.g. 0.3 ) produced fewer less optimal structures (within a maximum energy difference of $10 \%$ ). We speculate that this minor parameter difference may be due to the precise fitting achieved between experimental and control data sets by the automated FAST algorithm. FAST was written in ANSI C/C++ and is integrated into RNAstructure with FAST, which requires MFC (Microsoft Foundation Classes). RNA structures were drawn and colored using RNAViz $2^{67}$ and finalized in Adobe Illustrator.

\section{PSL2 Construct design, RNA synthesis and chemical modification for Mutate-and-Map}

Experiments. Double-stranded DNA templates were prepared by PCR assembly of DNA

13 oligomers designed by an automated MATLAB script as previously described (NA_Thermo,

14 available at https://github.com/DasLab/NA thermo $)^{68}$. Constructs for mutate-and-map $\left(\mathrm{M}^{2}\right)$

15 includes all single mutants to Watson-Crick counterpart. Compensatory mutants for mutation/rescue were designed based on base-pairing in the proposed secondary structure ${ }^{23}$. In

17 vitro transcription reactions, RNA purification and quantification steps were as described

18 previously ${ }^{68}$. One-dimensional chemical mapping, mutate-and-map $\left(\mathrm{M}^{2}\right)$, and mutation/rescue

19 were carried out in 96-well format as described previously ${ }^{68-70}$. Briefly, RNA was heated up and

20 cooled to remove secondary structure heterogeneity; then folded properly and incubated with

21 SHAPE reagent $(5 \mathrm{mg} / \mathrm{mL} \text { 1-methyl-7-nitroisatoic anhydride (1M7) })^{71}$; modification reaction was quenched and RNA were recovered by poly(dT) magnetic beads (Ambion) and FAM- 
$1 \mathrm{ddH}_{2} \mathrm{O}$; followed by reverse transcription to cDNA and heated $\mathrm{NaOH}$ treatment to remove RNA.

2 Final cDNA library was recovered by magnetic bead separation, rinsed, eluted in Hi-Di

3 formamide (Applied Biosystems) with ROX-350 ladder, loaded to capillary electrophoresis sequencer (ABI3100). Data processing, structural modeling, and data deposition: The HiTRACE software package version 2.0 was used to analyze CE data (both MATLAB toolbox and web server available ${ }^{72,73}$. Trace alignment, baseline subtraction, sequence assignment, profile fitting, attenuation correction and normalization were accomplished as previously described ${ }^{74,75}$.

Sequence assignment was accomplished manually with verification from sequencing ladders.

Data-driven secondary structure models were obtained using the Fold program of the RNAstructure package version $5.4^{76}$ with pseudo-energy slope and intercept parameters of 2.6 $\mathrm{kcal} / \mathrm{mol}$ and $-0.8 \mathrm{kcal} / \mathrm{mol}$. 2-dimensional $\mathrm{Z}$ score matrices for $\mathrm{M}^{2}$ datasets, and helix-wise bootstrapping confidence values were calculated as described previously ${ }^{23,68} . \mathrm{Z}$ score matrices were used as base-pair-wise pseudofree energies with a slope and intercept of $1.0 \mathrm{kcal} / \mathrm{mol}$ and 0 $\mathrm{kcal} / \mathrm{mol}$. Secondary structure images were generated by VARNA ${ }^{77}$. All chemical mapping datasets, including one-dimensional mapping, mutate-and-map, and mutation/rescue, have been deposited at the RNA Mapping Database (http://rmdb.stanford.edu $)^{78}$, accession codes: PSL2IAV_1M7_0001, PSL2IAV_RSQ_0001.

SHAPE analysis of LNA-targeted vRNA. A truncated DNA template of PR8 virus segment PB2 containing nucleotides 1-88 was prepared by PCR assembly of DNA oligomers, and in vitro transcription reactions, RNA purification and quantification steps were as described previously ${ }^{68}$. One-dimensional SHAPE chemical mapping was performed in 96-well plate format as described above with the following exception: once RNA was denatured and refolded as described, 100 $\mathrm{nM}$ of each prepared LNA was added to the folded RNA and incubated with $5 \mathrm{mg} / \mathrm{mL}$ of 
1 SHAPE reagent 1M7 (1-methyl-7-nitroisatoic anhydride). Modification quenching, RNA recovery, re-suspension, reverse transcription, cDNA sequencing and data processing were

3 performed as described in ref. 44.

4 Statistical analyses. We expressed the data as the mean \pm s.d. We used Student's $t$-test (to 5 compare two samples) or ANOVA (to compare multiple samples) (GraphPad InStat 3) for

6 statistical analysis. We performed the Kaplan-Meier log-rank test for survival analyses. We $7 \quad$ considered all $P$ values $>0.05$ not to be significant. 


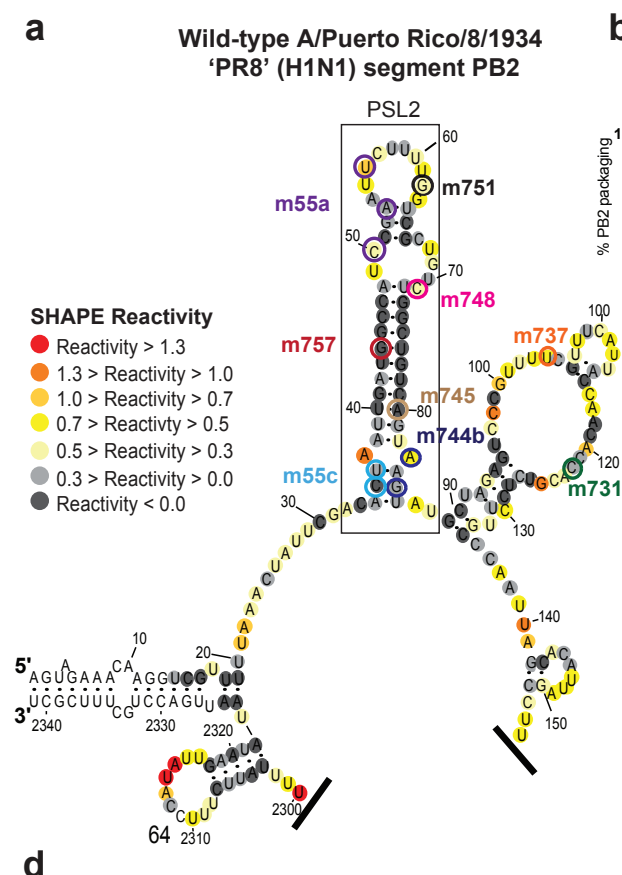

b $\quad{ }^{*} p<0.001 \quad$ C $m 757(G 44 C)$

Sequence conservation of PSL2 region

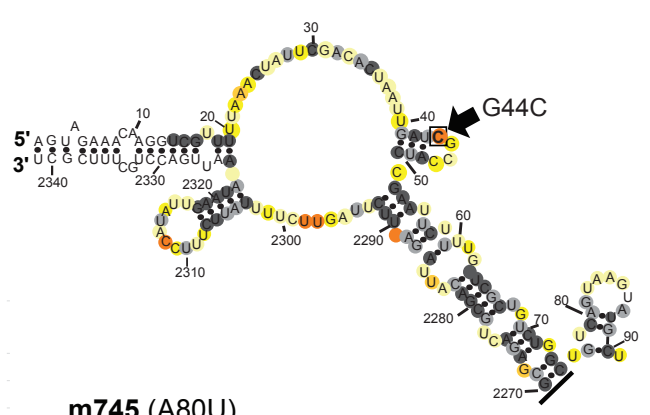

\begin{tabular}{|c|c|}
\hline $\mathrm{m} 757$ & $\mathrm{G} 44 \mathrm{C}$ \\
\hline $\mathrm{m} 55 \mathrm{a}$ & $\mathrm{CAU} 50,53,56$ \\
\hline $\mathrm{m} 751$ & $\mathrm{G} 62 \mathrm{C}$ \\
\hline $\mathrm{m} 748$ & $\mathrm{C} 71 \mathrm{U}$ \\
\hline $\mathrm{m} 745$ & $\mathrm{~A} 80 \mathrm{U}$ \\
\hline $\mathrm{m} 744 \mathrm{~b}$ & $\mathrm{AG} 83,85 \mathrm{U} A$ \\
\hline $\mathrm{m} 737$ & $\mathrm{U} 104 \mathrm{~A}$ \\
\hline $\mathrm{m} 731$ & $\mathrm{C} 122 \mathrm{G}$ \\
\hline
\end{tabular}

m745 (A80U)
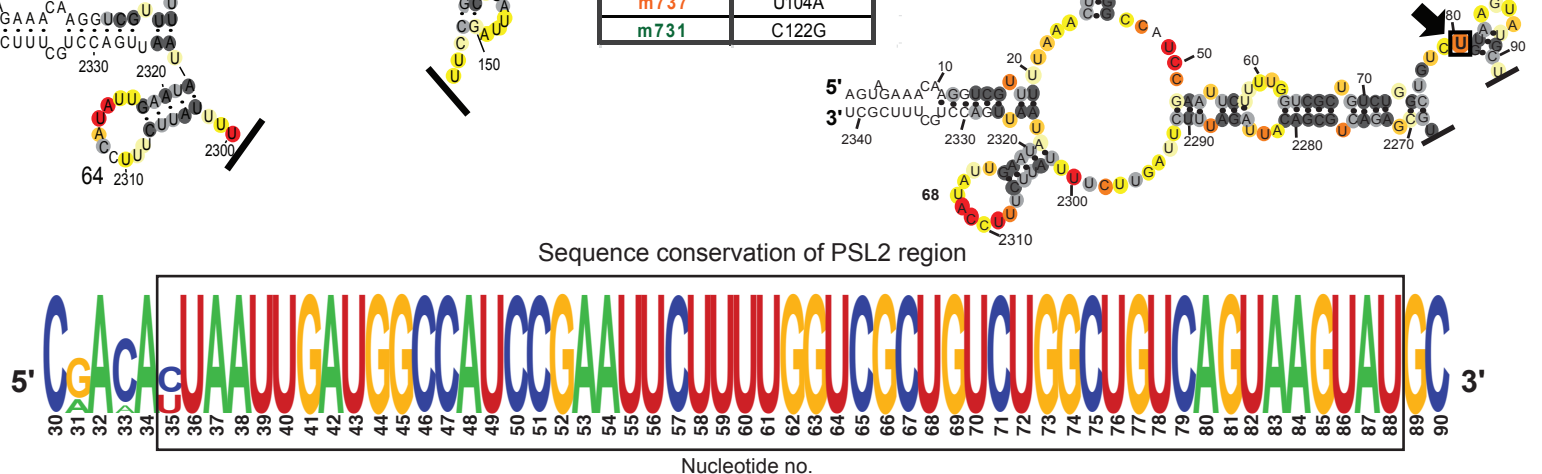

e
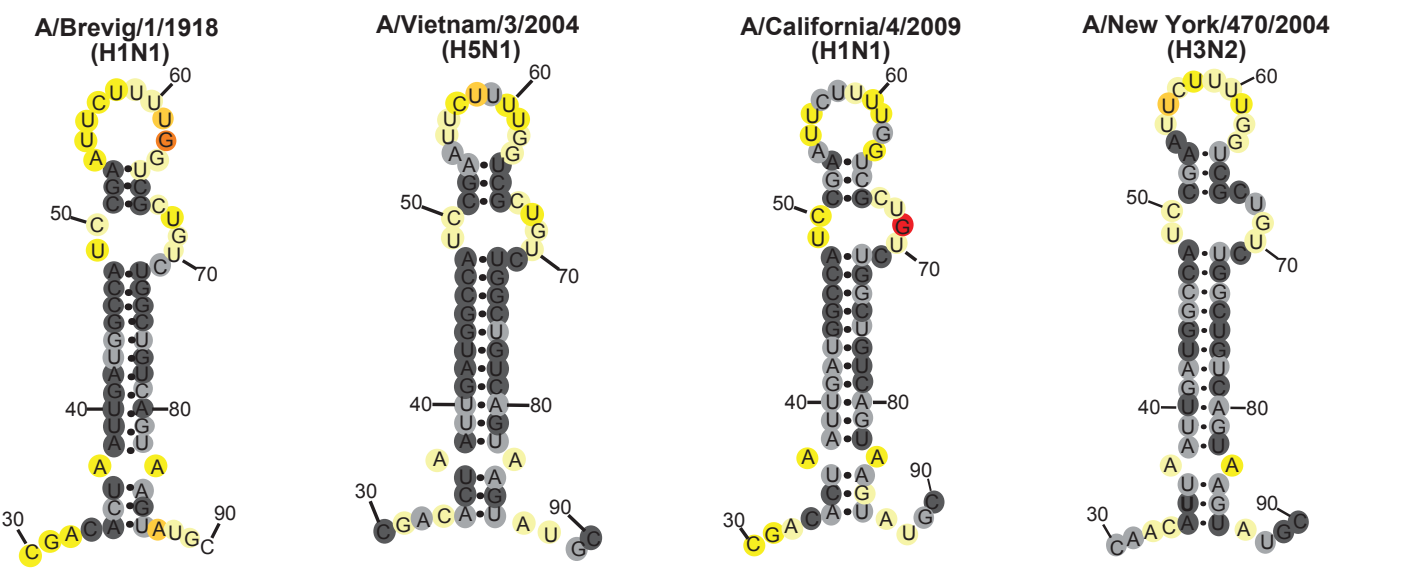

Figure 1. SHAPE-determined RNA secondary structures of wild-type PB2 and packaging mutant

vRNAs. SHAPE-chemical mapping performed on full-length (-)-sense PB2 vRNAs. Colors denote SHAPE reactivity, which is proportional to the probability that a nucleotide is single-stranded. All structures are truncated to highlight the $5^{\prime}$ termini sequence structure. (a) SHAPE-predicted wild-type PB2 RNA secondary structure from strain A/Puerto Rico/8/1934 "PR8” (H1N1). Color-coded circles 
1 correspond to nucleotides sites where synonymous mutations were reported to affect PB2 packaging ${ }^{13,16}$.

2 (b) Packaging efficiency of synonymous mutants in (a), determined by qPCR. Results representative of

3 two independent experiments with biological replicates, each performed in triplicate. Statistical analysis

4 was performed using one-way ANOVA with Dunnett's multiple comparisons test against the WT mean

5 by GraphPad Prism software. Error bars represents \pm standard deviation (s.d.). Box below indicates mutant name and corresponding nucleotide change. Nucleotide numbering shown in the genomic (-)sense orientation. (c) SHAPE-determined structures of PB2 packaging-defective mutant vRNAs, m757 (G44C) and m745 (A80U) indicating loss of PSL2's RNA secondary structure. Black arrowheads and boxed nucleotides denote site(s) of synonymous mutation. (d) Web-logo representation of the PSL2 region conservation across IAV strains and diverse influenza A viral subtypes (weblogo.berkeley.edu). The overall height represents sequence conservation at that nucleotide position, while the height of symbols within each position indicates the relative frequency of each nucleotide at that site. Black box denotes PSL2 region. Sequences included in the alignment: pandemic A/Brevig Mission/1/1918 (H1N1), pandemic A/California/04/2009 (H1N1), seasonal human A/New York/470/2004 (H3N2), A/Puerto Rico/8/1934 (H1N1), high pathogenic avian A/Vietnam/03/2004 (H5N1), avian A/mallard/Maryland/14OS1154/2014 (H6N1), pandemic A/Hong Kong/8/1968 (H3N2), and seasonal human A/New York/312/2001 (H1N1) (see Supplementary Fig. 1c). RNA nucleotides are numbered in ()-sense orientation. (e) SHAPE-determined structures of wild-type PB2 vRNA from pandemic and highly pathogenic strains, including different subtypes to modern human strains: 1918 pandemic (A/Brevig Mission/1/1918 (H1N1)), highly-pathogenic avian (A/Vietnam/1203/2004 (H5N1)), 2009 pandemic ‘swine' (A/California/04/2009 (H1N1)), and Fujian-like human seasonal virus, A/New York/470/2004 (H3N2). 
bioRxiv preprint doi: https://doi.org/10.1101/2021.08.21.457170; this version posted August 21, 2021. The copyright holder for this preprint (which was not certified by peer review) is the author/funder. All rights reserved. No reuse allowed without permission.

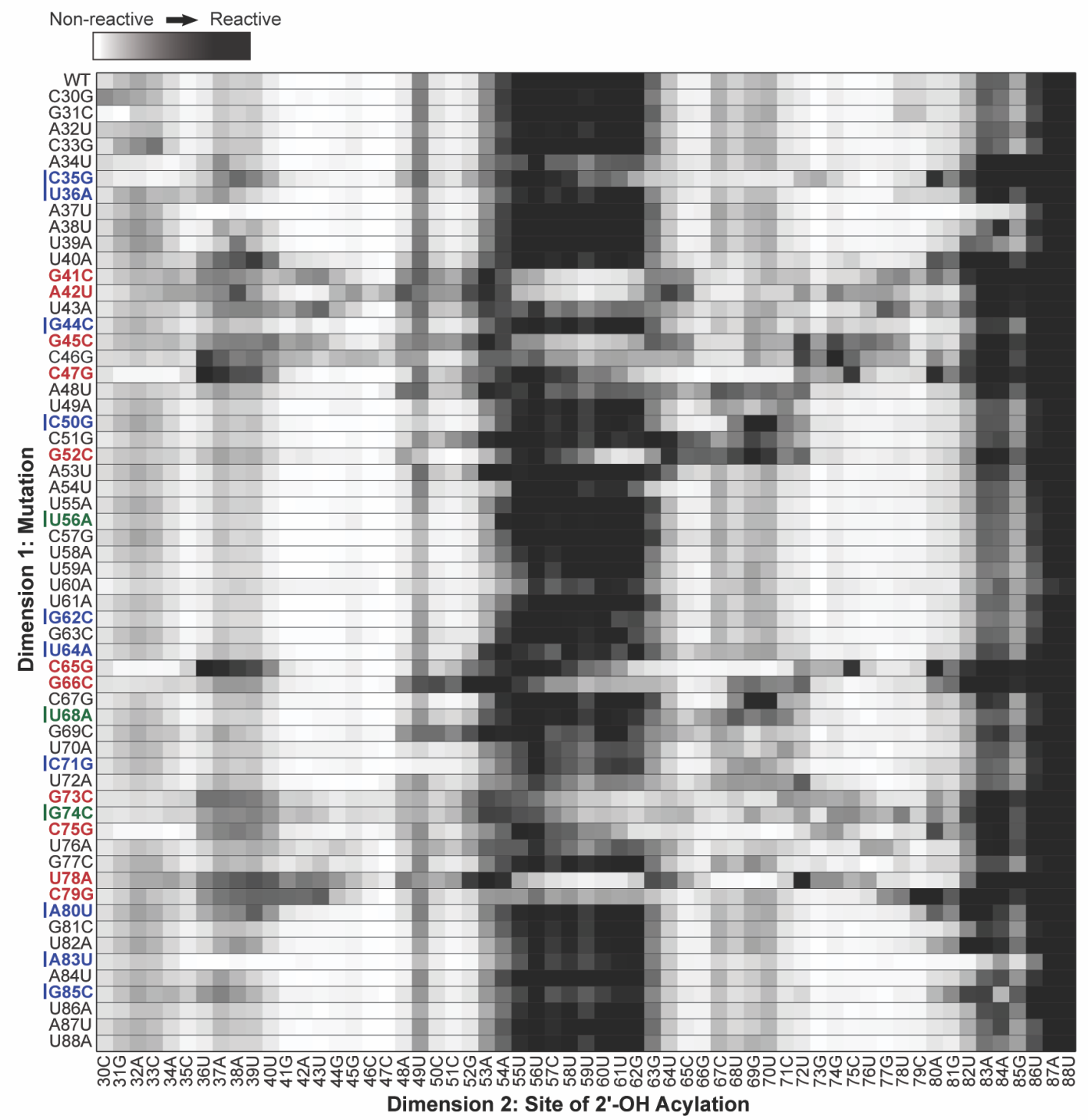

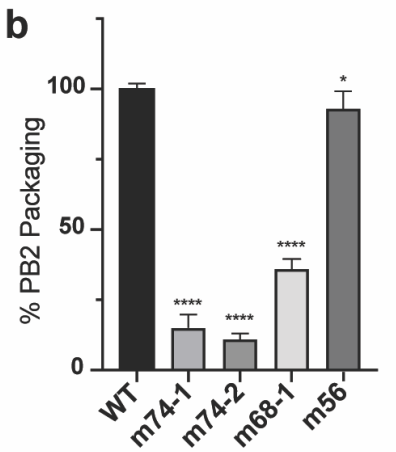

Syn. m74-1 (G74A)

Syn. m74-2 (UCG74AGC)

Syn. m68 (U68A) Syn. $\mathrm{m} 56$ (U56G)
C

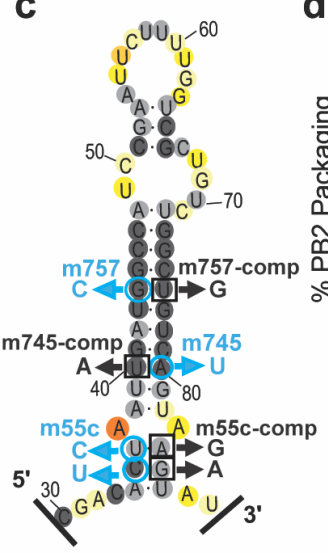

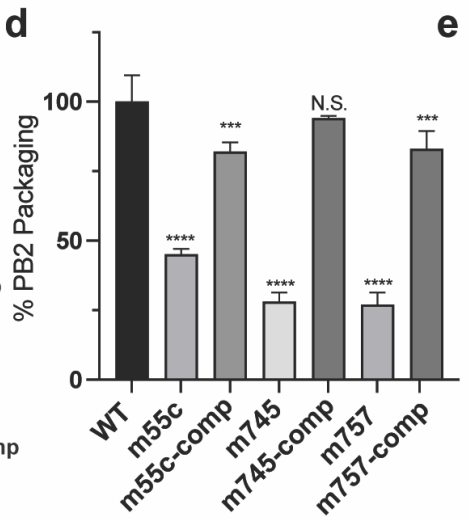

e 
1 Figure 2. 2-Dimensional Mutate-and-Map $\left(\mathrm{M}^{2}\right)$ analysis and empiric validation of PSL2 motif. (a)

2 Systematic single nucleotide mutation and mapping of resulting chemical accessibility reveals

3 interactions in the three-dimensional structure of the RNA. Chemical accessibilities, plotted in grayscale

4 (black, highest SHAPE reactivity), across 59 single mutations at single-nucleotide resolutions of PSL2

5 element from PR8 strain segment PB2. Reactivity peaks (left to right) correspond to nucleotides from the $5^{\prime}$ to $3^{\prime}$ end of the PB2 RNA. Nucleotides corresponding to known packaging mutation sites (ref. 13) are indicated on left in blue. Red bolded mutations denote prominent packaging-defective mutant sites predicted by $\mathrm{M}^{2}$ analysis. Green bolded mutations indicate synonymous mutant sites analyzed in (b). (b) Packaging efficiencies of $\mathrm{M}^{2}$-identified synonymous mutants read out by qPCR. Packaging efficiency represents the percentage of mutant PB2 vRNA packaging relative to parental wild-type PB2. Results from two independent experiments in biological duplicate, performed in technical triplicate $(\mathrm{n}=4)$.

12 Statistical analysis performed by ordinary one-way ANOVA using Dunnett's multiple comparisons test

13 against WT computed in GraphPad Prism software. (c) Previously described synonymous mutants (m757, $14 \mathrm{~m} 745, \mathrm{~m} 55 \mathrm{c})$ are mapped onto PSL2 structure. Compensatory, non-synonymous mutations m55c-comp, m745-comp, and m757-comp were designed at sites predicted to restore wild-type PSL2 structure based on SHAPE and mutate-and-map chemical analyses. Black boxed nucleotides denote site of compensatory mutation. (-)-sense vRNA orientation is shown. (d) Packaging efficiencies of packaging-defective and compensatory PB2 mutant viruses. For compensatory mutations where a non-synonymous change was required, a wild-type PB2 protein expression plasmid was co-transfected during virus rescue. Values given as percentage of PB2 vRNA packaging in comparison to wild-type parental PR8 virus. Results

21 from three independent experiments, assays performed in triplicate. (e) Virus titer determined by plaque 22 assay. Results in PFU / mL, plaque assays performed in triplicate. All error bars represent \pm s.d. Statistical 23 analyses in (d-e) performed as stated in (b) above. $* \mathrm{p}<0.05 * * \mathrm{p}<0.01 ; * * * \mathrm{p}<0.001 ; * * * * \mathrm{p}<0.0001$. 

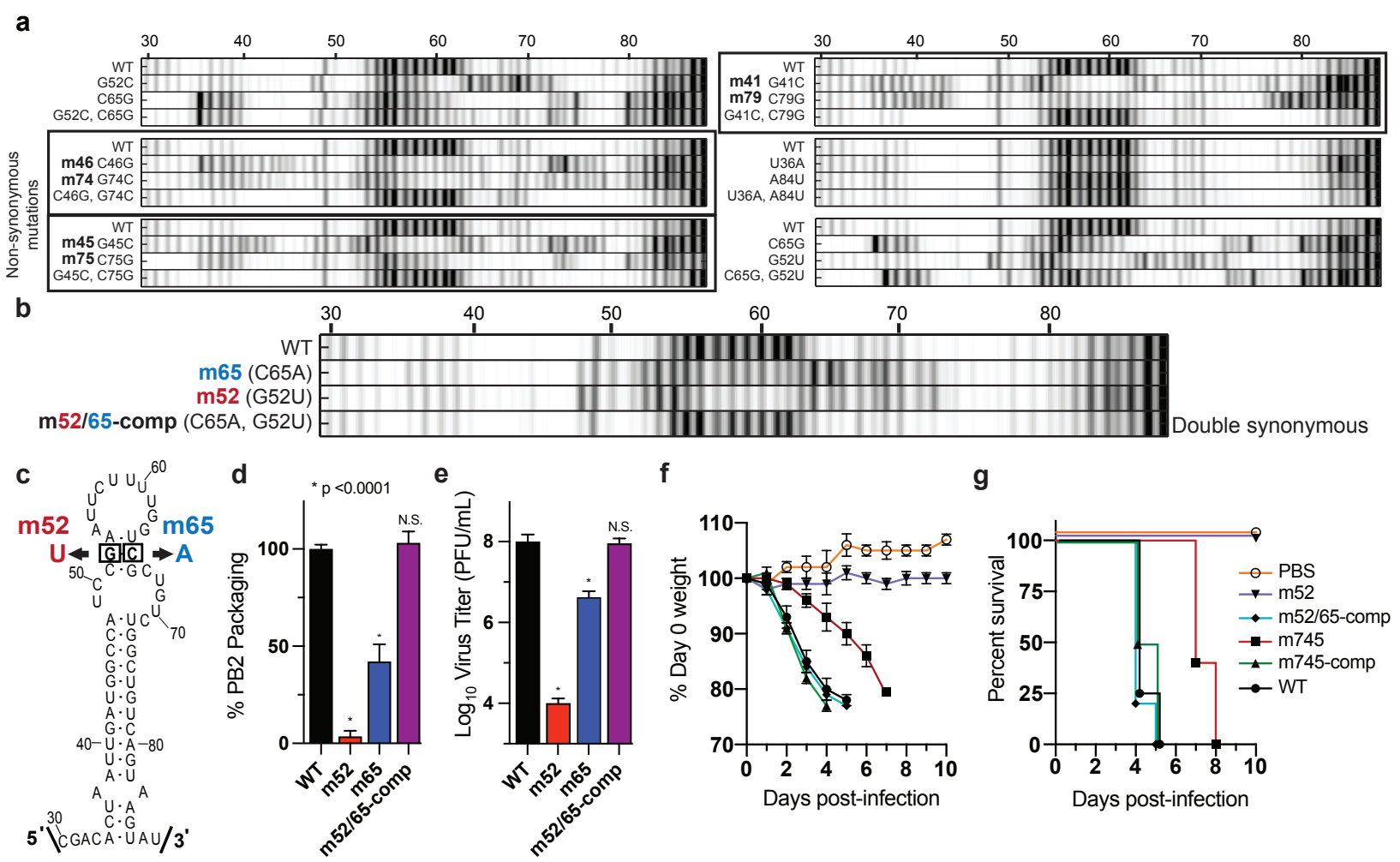

g

Figure 3. Mutate-Map-Rescue analysis reveals novel PB2 packaging-defective and compensatory

mutant partners. (a) Electropherograms from systematic single nucleotide mutation SHAPE chemical mapping with rescue (Mutate-Map-Rescue) ${ }^{21}$ analysis of individual and compensatory double mutations to test base-pairings from 1D-data-guided models and to identify predicted successful synonymous PSL2defective and compensatory mutant pairs. Chemical accessibilities, plotted in grayscale (black = highest SHAPE reactivity), across 59 single mutations at single-nucleotide resolutions of PSL2 element from PR8 strain segment PB2. Reactivity peaks (left to right) correspond to nucleotides from the $5^{\prime}$ to $3^{\prime}$ end of the PB2 RNA. See Supplementary Fig. 7 for complete set of Mutate-Rescue pairs. (b) Electropherogram of successful double synonymous mutant pair determined by Mutate-Map-Rescue analysis. (c) Mutational design of single mutants $\mathrm{m} 52(\mathrm{G} 52 \mathrm{U})$ and $\mathrm{m} 65$ (C65A), and the double m52/65-comp rescue pair on the PSL2 structure. (d) Packaging efficiency of the synonymous single and double mutant Mutate-Rescue pair. Values given as a percentage of PB2 vRNA packaging relative to WT parental PR8 virus. Results represent two independent experiments with biological replicates and performed in technical triplicate $(n=4)$ except for the m65 mutant, which were performed in biological triplicate $(n=6)$. (e) Viral titer of the 
1 supernatants collected in (d) in PFU / mL, plaque assay results in triplicate. Statistical analysis performed

2 in (d-e) by ordinary one-way ANOVA using Dunnett's multiple comparisons test against WT using

3 GraphPad Prism software. * $\mathrm{p}<0.0001$, N.S. = not significant. (f-g) Percent Day 0 weight and survival of

4 mice infected with single PSL2-disrupting, and compensatory PSL2-restoring double-mutant viruses. Six

5 to eight weeks old BALB/c female mice ( $\mathrm{n}=6$ mice / group) were intranasally infected with PR 8 wild-

6 type (WT) virus, packaging-defective single mutant viruses, m52 and m745, compensatory double mutant

7 viruses, m52/65-comp and m745-comp, or PBS control. (f) Percent Day 0 weight. (g) Kaplan-Meier

8 survival plot of the individual cohorts depicted in (f). All error bars represent \pm s.d. 
a

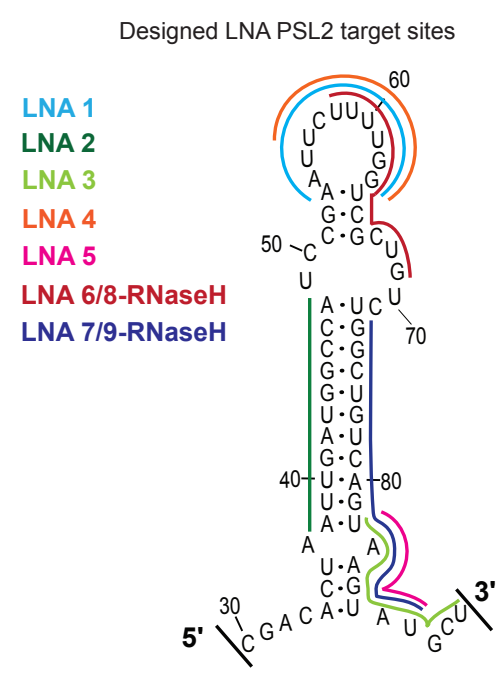

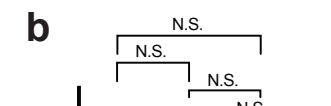

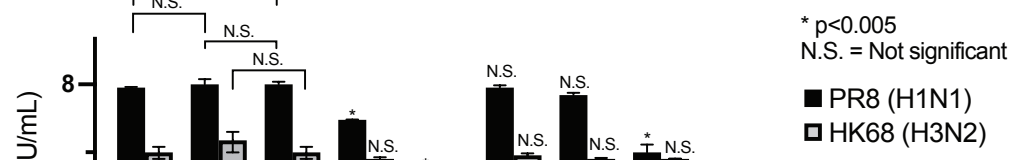

C

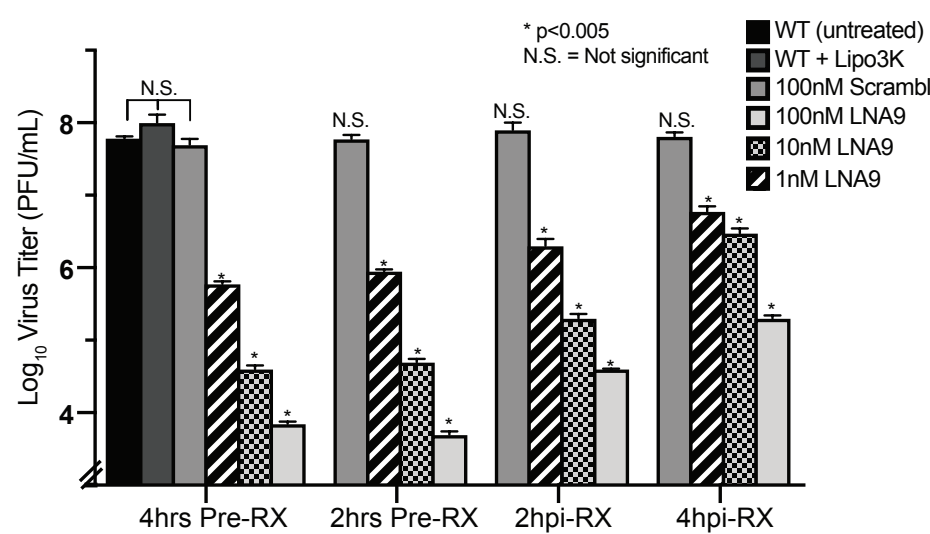

e

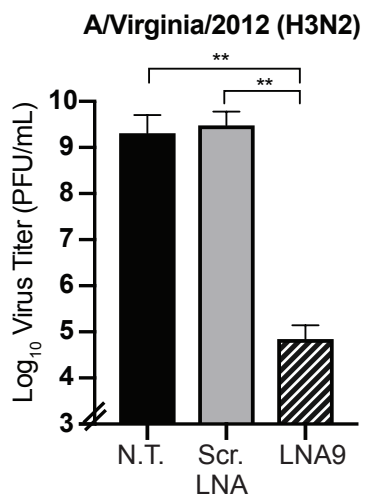

A/California/2009 (pH1N1)

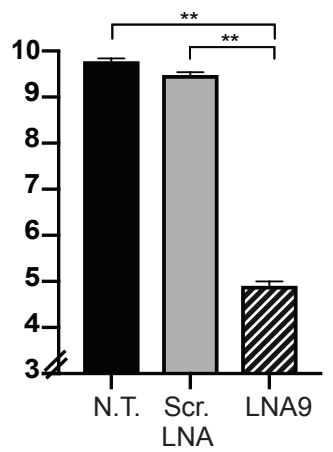

A/PR8/1934 (H1N1)

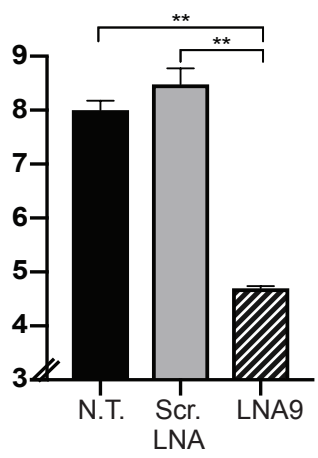

A/HK/1968 (H3N2)

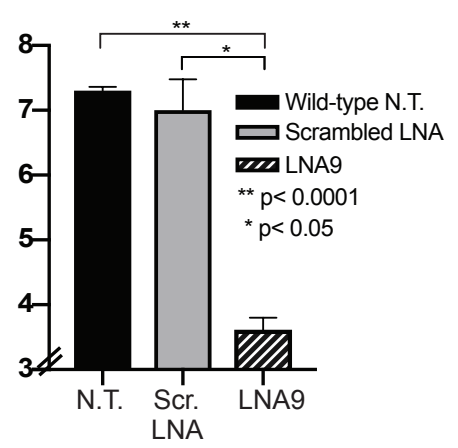

Figure 4. Locked Nucleic Acids targeting PSL2 RNA structure display potent antiviral activity in vitro. (a) Location of complementary Locked Nucleic Acids (LNAs) designed against different regions of the PSL2 structure. (b) LNA antiviral screen: MDCK cells were pretreated with $100 \mathrm{nM}$ of each

5 designated LNA or a scrambled mismatch LNA for 4 hours prior to infection with PR8 (H1N1) virus or 
1 A/Hong Kong/8/68 (H3N2) virus (0.01 MOI). 48 hours post-infection (hpi), supernatant was collected,

2 and viral titers determined by plaque assay. Assays performed in biological triplicate with three technical

3 replicates $(\mathrm{n}=9) . *$ Statistical significance shown between non-treated WT + Lipo and LNA-treated

4 samples, unless otherwise indicated. (c) Time course of pre-treatment (Pre-RX) versus post-infection

5 treatment with LNA9 at titrating concentrations $(100 \mathrm{nM}, 10 \mathrm{nM}, 1 \mathrm{nM}) . \mathrm{WT}+\mathrm{Lipo} 3 \mathrm{k}=$ non-treated

6 infection with Lipofectamine 3000 control. Pre-RX: MDCK cells were treated with LNA9 or Scrambled

$7 \quad$ LNA either 2 or 4 hours prior to infection with PR8 virus at an MOI of 0.01 . Post-infection treatment: MDCK cells were infected with PR8 virus at an MOI of 0.01 and treated with LNA9 or Scrambled LNA at either 2 or 4 hpi. Supernatant was collected $48 \mathrm{hpi}$, and viral titers determined by plaque assay in biological and technical triplicate $(\mathrm{n}=9)$. * Significance determined relative to WT PR8-Lipo. (d)

11 Packaging efficiency of PB2 vRNA from PR8 viruses treated with 100 nM LNA9 or scrambled LNA

12 control. Values given as a percentage of PB2 vRNA packaging in comparison to non-treated wild-type

13 PR8 virus, readout by qPCR. Results from two biological replicates, assays performed in technical

14 triplicates $(\mathrm{n}=6)$. (e) LNA9 antiviral efficacy against multiple influenza A strains: A/Virginia/2012

15 (H3N2), A/California/2009 (pH1N1), A/PR8/1934 (H1N1), and A/Hong Kong/1968 (H3N2). MDCK

16 cells were pretreated with $100 \mathrm{nM}$ of LNA9 or Scramble 12 hours prior to infection with the indicated

17 viruses at an MOI of 0.01. Plaque assays performed in biological and technical triplicate $(\mathrm{n}=9)$. All error

18 bars represent \pm s.d. 
a

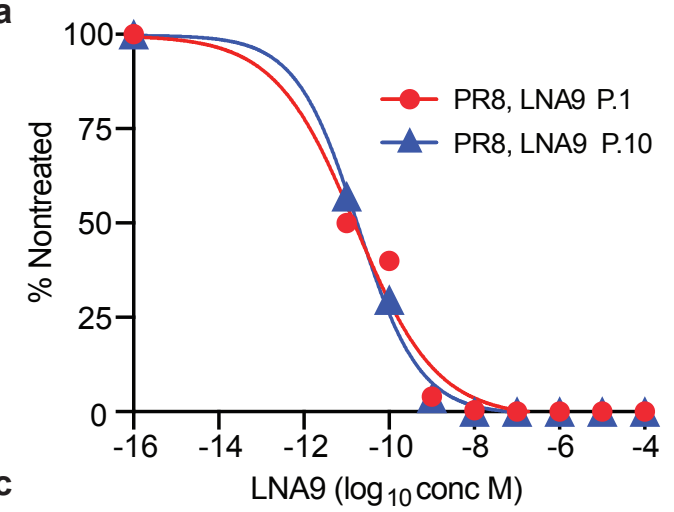

C

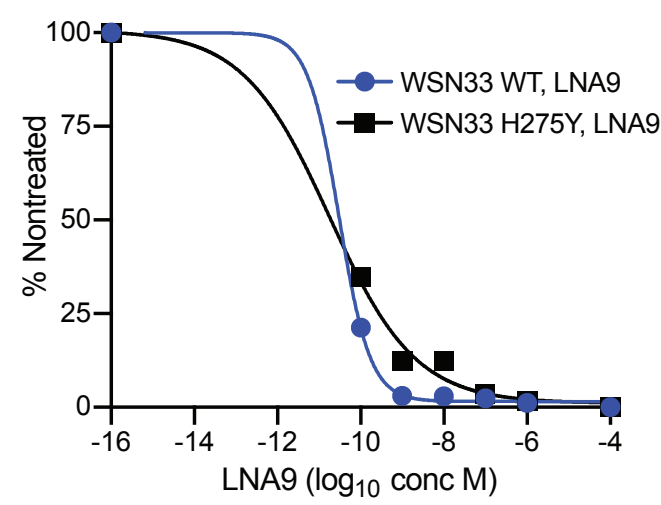

b
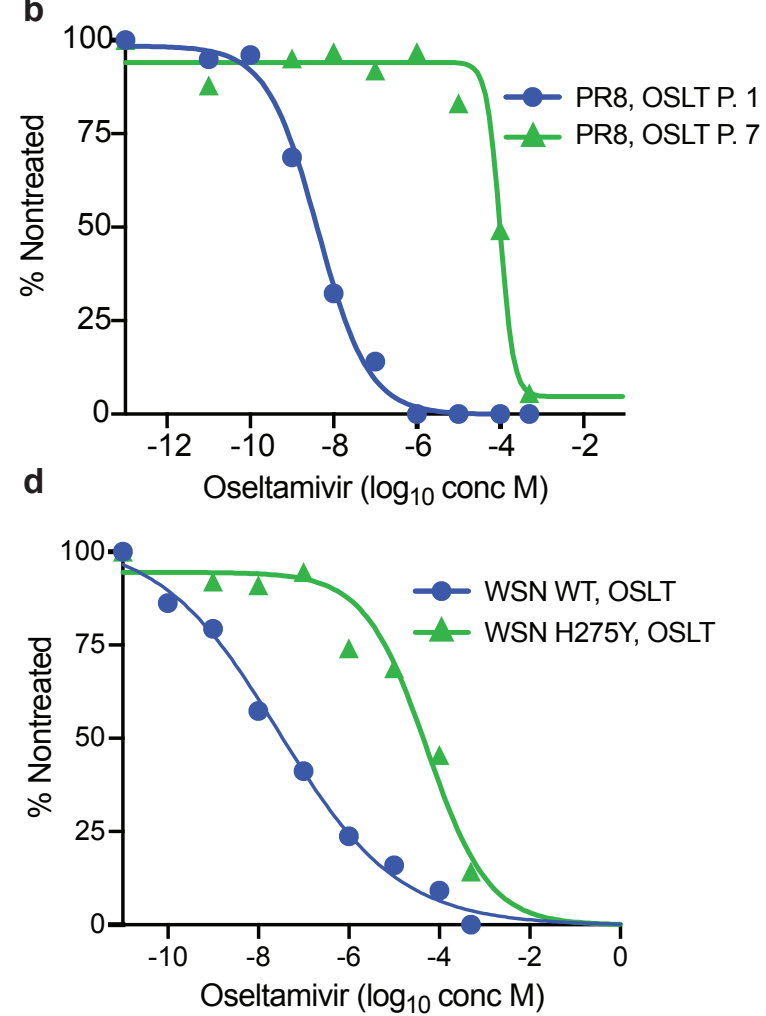

e

In vitro selection and characterization of influenza A variants in response to oseltamivir carboxylate and LNA9 drug treatment

\begin{tabular}{lccc}
\multicolumn{1}{c}{ Virus } & Passage & $\begin{array}{c}\text { Oseltamivir } \\
\text { EC 50 (nM) }\end{array}$ & $\begin{array}{c}\text { LNA9 } \\
\text { EC 50 (nM) }\end{array}$ \\
\hline A/PR8/34 (H1N1) "PR8" & 1 & 4.2 & 0.016 \\
& 7 & 99,530 & - \\
A/WS/33 (H1N1) "WSN" WT & 10 & -- & 0.022 \\
A/WS/33 (H1N1) "WSN" H274Y & & 28 & 0.03 \\
\hline
\end{tabular}

Figure 5. In vitro selection and sensitivity of passaged virus in response to drug treatment. (a,b)

Wild-type PR8 virus was serially passaged in the presence of either LNA9 (a) or oseltamivir carboxylate

(b) over time with escalating concentrations of drug. Viral supernatant from each passage was collected and titered by plaque assay. (a) LNA9: MDCK cells were pretreated with varying concentrations of LNA9 12 hours prior to infection at $0.01 \mathrm{MOI}$ of passage 1 (P.1) or passage 10 (P.10) LNA9-treated PR8 virus. After 48 hours, viral supernatant was collected and titered for each drug dilution. Results expressed as a percentage of nontreated virus titer. The drug concentration that caused $50 \%$ decrease in the percent 
1 of PFU titer in comparison to untreated controls was defined as the $\mathrm{EC}_{50}$. (b) Oseltamivir: Confluent

2 MDCK cells were infected with 100 PFU of passage 1 (P.1) or passage 7 (P.7) OSLT-treated PR8 virus

3 and drug sensitivity was determined by plaque reduction assay. The number of viral plaques with each

4 drug concentration was counted and plaque number was normalized against the nontreated control to

5 determine the $\mathrm{EC}_{50}$. (c,d) In vitro sensitivity of wild-type WSN33 (H1N1) virus and NAI-resistant WSN

6 H275Y NA mutant virus to LNA9 (c) or oseltamivir carboxylate (d). (e) Summary table of EC 50 values

7 from graphs (a-d). 
a

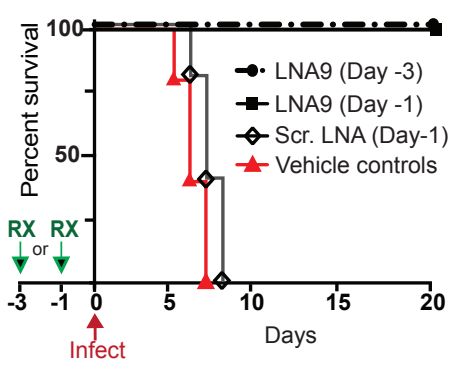

e

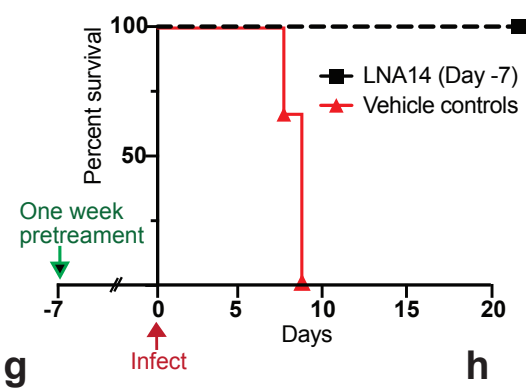

b

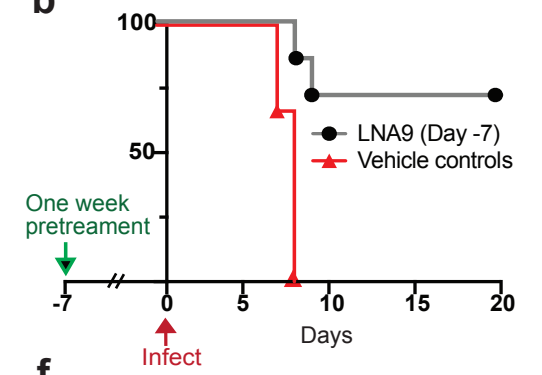

f

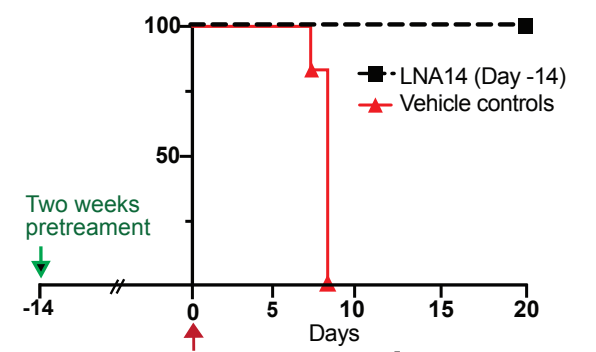

C

c

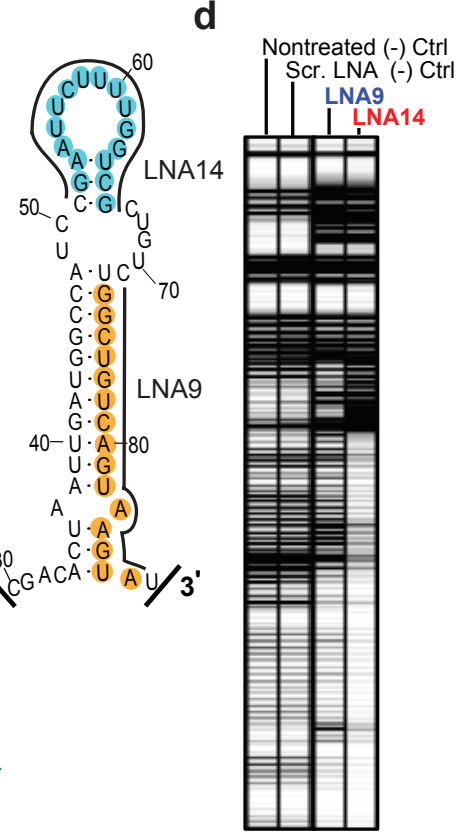

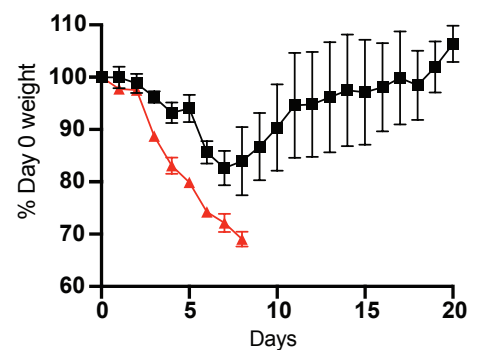

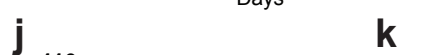

k

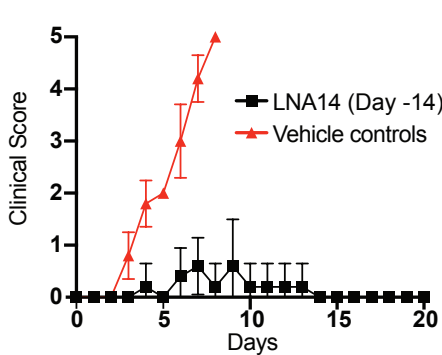

I

Single-dose LNA14
treatment

Cos

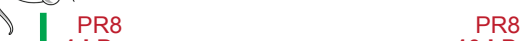

$\checkmark 1 \mathrm{LD}_{100}$
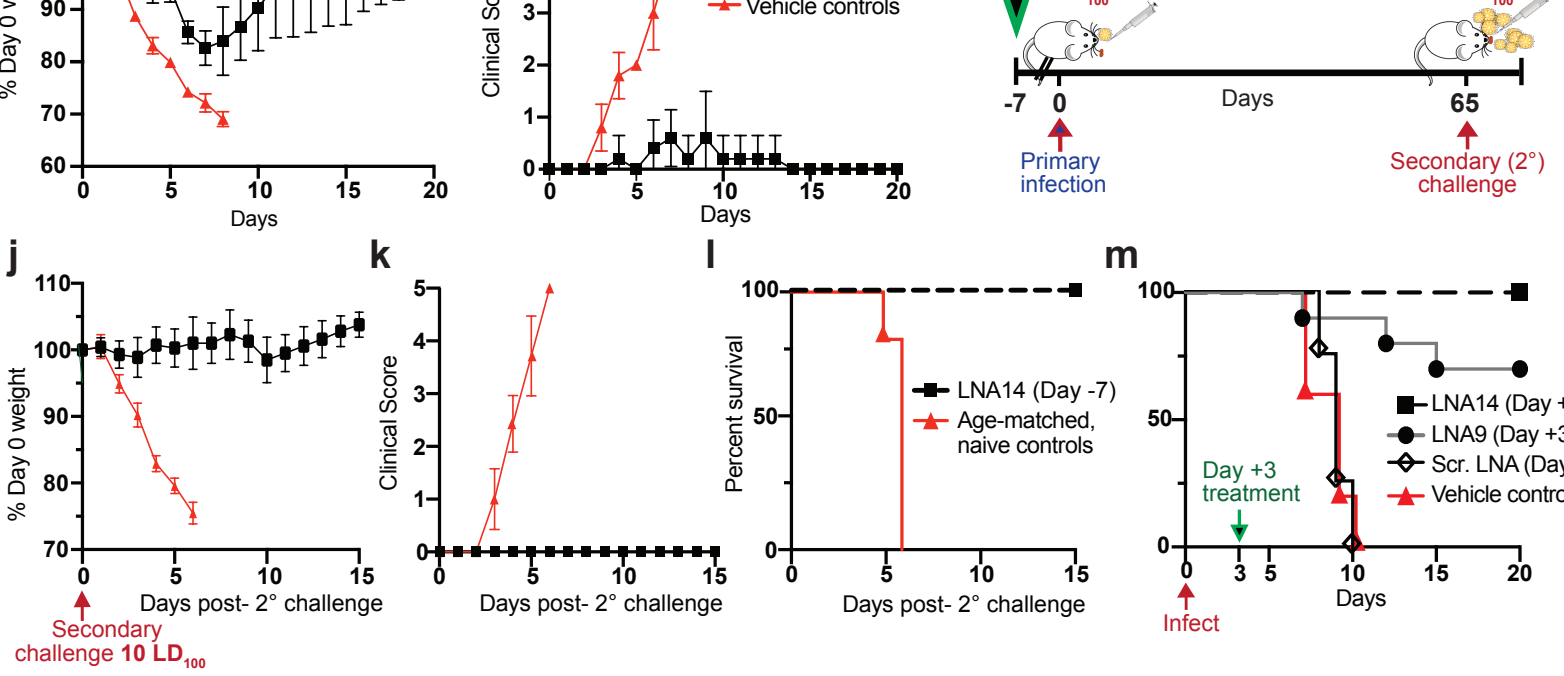

m

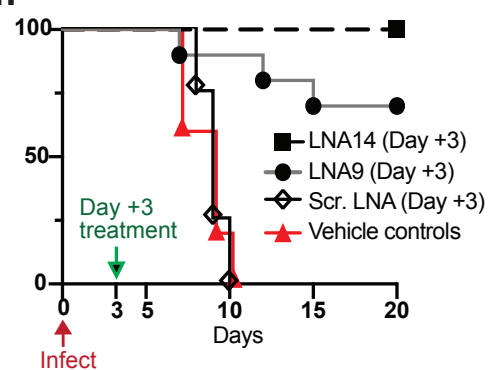

Figure 6. PSL2-targeted LNAs demonstrate potent antiviral activity in vivo. (a-b) Effect of intranasal

3 LNA prophylactic treatment on survival of virus-infected mice. Kaplan-Meier survival plots. Mice $(\mathrm{n}=7$ mice/group) were intranasally administered a single dose of LNA9, Scrambled LNA, or Vehicle (mocktreated) followed by a lethal inoculum of wild-type PR8 virus (a) dosed with $20 \mu \mathrm{g}$ LNA 3 days before infection (Day -3) or 1 day before infection (Day -1); (b) one week pretreatment with a single $30 \mu \mathrm{g}$ dose of LNA9 or vehicle control. (c) Target sites of LNA9 and newly designed, LNA14, mapped to the PSL2 
1 structure. (d) Electrophoretic profile of SHAPE analysis performed on non-treated, Scrambled LNA,

2 LNA9, and LNA14 at $100 \mathrm{nM}$ concentration in the presence of PR8 PB2 vRNA. Labeling with 1M7

3 SHAPE reagent shown. (e) Kaplan-Meier survival plot of mice ( $\mathrm{n}=7$ mice/group) intranasally pretreated

4 with a single dose of $30 \mu \mathrm{g}$ LNA14 or vehicle control one week (Day -7) before lethal PR8 infection. (f-

5 h) A single dose of $40 \mu \mathrm{g}$ LNA14 or vehicle was I.N. administered two weeks (Day -14) before PR8 virus

6 infection. (f) Kaplan-Meier survival plot. (g) Percent day 0 weight of mice. (h) Clinical score. (i-l) Mice

$7 \quad(\mathrm{n}=7)$ were given a single $40 \mu \mathrm{g}$ intranasal dose of LNA14 one week prior to a primary lethal infection at

$81 \mathrm{LD}_{100}$ of PR8 virus (e). Sixty-five days post-initial infection, surviving mice from (e) along with age-

9 matched naïve controls ( $\mathrm{n}=7$ /group) were challenged a second time at $10 \mathrm{LD}_{100}$. (i) Challenge study

10 timeline. (j) Percent day 0 weight of mice. (k) Clinical score. (l) Kaplan-Meier survival curve. (m) Mice

11 ( $\mathrm{n}=10 /$ group) were infected with a lethal dose of PR8 wild-type virus. Three days post-infection, mice

12 were given a single dose of $40 \mu$ g of LNA14, LNA9, Scrambled LNA, or vehicle control by I.V.

13 injection. Kaplan-Meier survival plot. 